\title{
PULSATING B-TYPE STARS IN THE OPEN CLUSTER NGC 884: FREQUENCIES, MODE IDENTIFICATION, AND ASTEROSEISMOLOGY
}

\author{
S. Saesen ${ }^{1,2}$, M. Briquet ${ }^{2,3,6}$, C. Aerts ${ }^{2,4}$, A. Miglio ${ }^{5}$, and F. Carrier ${ }^{2}$ \\ ${ }^{1}$ Observatoire de Genève, Université de Genève, Chemin des Maillettes 51, 1290 Sauverny, Switzerland; sophie.saesen@unige.ch \\ ${ }^{2}$ Instituut voor Sterrenkunde, Katholieke Universiteit Leuven, Celestijnenlaan 200 D, B-3001 Leuven, Belgium \\ ${ }^{3}$ Institut d'Astrophysique et de Géophysique de l'Université de Liège, Allée du 6 Août 17, B-4000 Liège, Belgium \\ ${ }^{4}$ Department of Astrophysics, Radboud University Nijmegen, P.O. Box 9010, 6500 GL Nijmegen, The Netherlands \\ ${ }^{5}$ School of Physics and Astronomy, University of Birmingham, Edgbaston, Birmingham, B15 2TT, UK \\ Received 2013 May 7; accepted 2013 June 30; published 2013 September 16
}

\begin{abstract}
Recent progress in the seismic interpretation of field $\beta$ Cep stars has resulted in improvements of the physical description in the stellar structure and evolution model computations of massive stars. Further asteroseismic constraints can be obtained from studying ensembles of stars in a young open cluster, which all have similar age, distance, and chemical composition. We present an observational asteroseismology study based on the discovery of numerous multi-periodic and mono-periodic B stars in the open cluster NGC 884. We describe a thorough investigation of the pulsational properties of all B-type stars in the cluster. Overall, our detailed frequency analysis resulted in 115 detected frequencies in 65 stars. We found 36 mono-periodic, 16 bi-periodic, 10 tri-periodic, and 2 quadru-periodic stars and one star with nine independent frequencies. We also derived the amplitudes and phases of all detected frequencies in the $U, B, V$, and $I$ filter, if available. We achieved unambiguous identifications of the mode degree for 12 of the detected frequencies in nine of the pulsators. Imposing the identified degrees and measured frequencies of the radial, dipole, and quadrupole modes of five pulsators led to a seismic cluster age estimate of $\log (\mathrm{age} / \mathrm{yr})=7.12-7.28$ from a comparison with stellar models. Our study is a proof-of-concept for and illustrates the current status of ensemble asteroseismology of a young open cluster.
\end{abstract}

Key words: open clusters and associations: individual (NGC 884) - stars: early-type - stars: oscillations - techniques: photometric

Online-only material: color figures, figure set, machine-readable and VO tables

\section{INTRODUCTION}

Main-sequence stars of spectral type $\mathrm{O} 9$ to $\mathrm{B} 2$ are very interesting targets for asteroseismology, i.e., the study of the internal stellar structure by interpreting the observed oscillation characteristics. Indeed, these stars have a convective core which strongly determines the evolution of the star, while they do not suffer from a strong stellar wind. Moreover, the internal rotation of these stars is not well known, but it causes mixing of the chemical elements which may also have an important effect on their evolution. And finally, as they are the progenitors of core-collapse supernovae, these stars will chemically enrich the universe.

In this paper, we present an observational asteroseismology study of pulsating B stars in the cluster NGC 884. This study is a particular observational part of a global photometric study of that cluster already introduced by Saesen et al. (2010) and can be seen as a multi-color photometric analogue of the recent spectroscopic study of B stars in the cluster by Marsh Boyer et al. (2012).

The simultaneous exploitation of oscillation frequencies of stars in the same environment obviously has advantages compared to the study of single stars. Imposing the same age and chemical composition will give much tighter constraints when interpreting the observed oscillation spectra. For this reason, various observational initiatives were taken in the past decade (e.g., Handler et al. 2008; Majewska et al. 2008; Michalska et al. 2009; Handler \& Meingast 2011; Jerzykiewicz et al. 2011, for recent

\footnotetext{
6 Postdoctoral Researcher of the Fund for Scientific Research, Fonds de la Recherche Scientifique-FNRS, Belgium.
}

examples of young open clusters with pulsating B stars). Based on observational results in the literature, the study by Balona et al. (1997) led to a derivation of a frequency - age - mass relation for three young open clusters, from averaging out detected but unidentified pulsation frequencies of cluster $\beta$ Cep stars and relying on evolutionary models with one fixed set of the initial hydrogen fraction and metallicity of $(X, Z)=(0.70,0.02)$, ignoring core overshooting (i.e., taking $\alpha_{\mathrm{ov}}=0.0$ ). Despite these restrictions, the authors came to the conclusion that such age estimation is already more precise than the one based on isochrone fitting and could be improved if the detected pulsation modes could be identified in the future.

Putting more efforts into ground-based young open cluster campaigns is still relevant, even with the enormous database now at hand gathered by the satellites CoRoT and Kepler. Indeed, Kepler did not reveal many early B stars with strong pulsations (Balona et al. 2011). Among 48 variable Kepler B-type stars, 15 are pulsating in the $g$-mode regime, of which 7 show weak frequencies in the $p$-mode range. These are not good candidates for asteroseismic studies, since the observations in white light and the lack of frequency splittings do not allow for mode identification. Moreover, their pulsation amplitudes are too low for ground-based follow-up studies. CoRoT did observe several pulsating B stars, but dedicated long-term ground-based photometry and time-resolved spectroscopy were necessary to perform stellar modeling (e.g., Degroote et al. 2009; Briquet et al. 2009; Aerts et al. 2011).

While asteroseismology of the few open clusters in the field of view of Kepler based on solar-like oscillations in red giants is progressing fast (Stello et al. 2011; Hekker et al. 2011; Miglio et al. 2012), leading to seismic constraints on mass loss on 
the red giant branch, it concerns clusters of a few Gyr old. Neither CoRoT nor Kepler is observing young open clusters with $\beta$ Cep pulsators. Therefore, our ground-based photometric study of the B-type stars in NGC 884 combined with their recent spectroscopic analogues (Strom et al. 2005; Huang \& Gies 2006; Marsh Boyer et al. 2012) offers an interesting and different approach to the advancement of understanding such young massive objects.

Saesen et al. (2010) presented a description of the global multi-site study of NGC 884 in terms of differential timeresolved multi-color CCD photometry of a selected field of the cluster. This study also contained a global stellar variability study among the 3165 cluster members through an automated frequency analysis in the $V$ filter, leading to the identification of 36 multi-periodic and 39 mono-periodic B stars, 19 multi-periodic and 24 mono-periodic A and F stars, and 20 multi-periodic and 20 mono-periodic variable stars of unknown nature. Moreover, 15 irregular variable stars were found, of which 6 are supergiant stars, 8 are Be stars, and 1 star is a B star with a similar light-curve behavior as the Be stars. Also 6 new and 4 candidate eclipsing binaries were detected, apart from 2 known cases.

The present paper exploits the full photometric multi-site campaign data set of the periodic B stars and couples them to published ground-based spectroscopy of those stars for a global interpretation in terms of variability. We present a detailed frequency analysis of all discovered periodic B stars using all filters. Moreover, we attempt to derive the mode degree $\ell$ of all the detected oscillation frequencies by means of the well-known method of amplitude ratios (e.g., Aerts et al. 2010, Chapter 6), making use of the multi-color photometric time series. Finally, we select the $\beta$ Cep stars in NGC 884 and use them to compare the observed properties of their oscillations with those predicted for models considering their measured rotational velocities. This allowed us to deduce a seismic age range for the cluster which is compatible with the one deduced previously from an eclipsing binary in the cluster.

\section{DATA DESCRIPTION}

For a detailed description of the extensive overall 12-site campaign, which resulted in almost 77,500 images and $92 \mathrm{hr}$ of photoelectric data in $U B V I$, spread over three observing seasons, we refer to Saesen et al. (2010). That paper also contains a comprehensive report of the calibration and reduction process, which is thus omitted here, but we recall that the resulting light curves of the brighter stars have a precision of 5.7 mmag in $V$, $6.9 \mathrm{mmag}$ in $B, 5.0 \mathrm{mmag}$ in $I$, and $5.3 \mathrm{mmag}$ in $U$. In the current paper, we focus exclusively on the variable B stars found in the cluster and perform the first detailed analyses of their light curves.

First, we manually checked the automated merging of the light curves of all sites, which are a combination of Johnson $(B, V, I)$, Bessell $(U, B, V, I)$, Geneva $(U, B, V)$, and Cousins $(I)$ filter systems, and we applied a magnitude shift where needed. Then, to avoid second-order extinction and seeing effects, we calculated the residuals of a preliminary harmonic solution, for which we did not include possibly spurious alias frequencies. For every observing site separately, we then fitted a linear function of the airmass to these residuals, subtracted it from these residuals, and finally fitted a linear function of the seeing, where we used the FWHM values of the star's point-spread function on the CCD as measure. Both linear trends were then removed from the original data. This led to better results than those from the automated procedures adopted in Saesen et al. (2010) as illustrated by the case of Oo 2444, for which the frequency close to 1 day $^{-1}$ does not occur any more (compare Figure 1 with Figure 11 of Saesen et al. 2010). Such manual corrections were applied for all B stars treated in this paper.

For some selected stars (Oo 2246, Oo 2299, Oo 2444, Oo 2488, Oo 2566, Oo 2572, and Oo 2649), we also assembled photoelectric data. Here, we merged them with the other $U B V I$ data sets in Saesen et al. (2010). Simultaneous Strömgren $u v b y$ and Geneva $U B_{1} B B_{2} V_{1} V G$ measurements of (suspected) $\beta$ Cep stars were collected using the Danish photometer at Observatorio Astronómico Nacional de San Pedro Mártir (OAN-SPM) and the P7 photometer at Observatorio del Roque de los Muchachos (ORM), respectively. The reduction method for the photoelectric data taken at OAN-SPM is described in Poretti \& Zerbi (1993) and references therein. For the reduction of the photoelectric measurements of ORM with P7, we refer to Rufener (1964, 1985). We combined the filters $U$ and $u, B$ and $v$, and $V$ and $y$, since the effective wavelengths of these filters are similar. We compared the photometric zero points and adjusted them if necessary. In the end, we obtained an ultraviolet, blue, visual, and near-infrared light curve, which we refer to as $U, B, V$, and $I$ hereafter.

\section{BASIC STELLAR PARAMETERS}

We obtained the basic stellar parameters, the effective temperature $T_{\text {eff }}$, and the luminosity $\log \left(L / L_{\odot}\right)$, from different sources. First, we have absolute photometry of NGC 884 in the seven Geneva filters at our disposal (see Section 7 of Saesen et al. (2010) for more information on the data and their reduction). The calibrations of the Geneva system allow a physically meaningful classification of early-type stars, by the determination of the stellar parameter $T_{\text {eff }}$, based on the code CALIB (Kunzli et al. 1997) and the estimates of the average values of the six Geneva colors $(U-B),\left(B_{1}-B\right),\left(B_{2}-B\right),\left(V_{1}-B\right),(V-B)$, and $(G-B)$. This calibration is based on the calculation of synthetic colors using Kurucz atmosphere models with solar metallicities. The effective temperature values and their interpolation errors in the grid of atmosphere models, as provided by CALIB, are presented in Table 1 . We adopted $1000 \mathrm{~K}$ on $T_{\text {eff }}$ as realistic $1 \sigma$ uncertainties including the systematic, statistical, and interpolation errors (Morel et al. 2006; De Cat et al. 2007), except if the interpolation error was larger.

To determine the luminosity of the stars on the basis of our absolute photometric measurements, we first calculated the interstellar reddening with the formula

$$
A_{V}=\left[3.3+0.28(B-V)_{\mathrm{J}, 0}+0.04 E(B-V)_{\mathrm{J}}\right] E(B-V)_{\mathrm{J}}
$$

(Arenou et al. 1992). For the reddening $E(B-V)_{\mathrm{J}}$ in the Johnson system, we used the non-uniform reddening values discussed in Section 7.2 of Saesen et al. (2010). These reddening values are around $E(B-V)_{\mathrm{J}}=0.53 \pm 0.05$, which is well in agreement with the recent literature values listed in Southworth et al. (2004a) and the value deduced by Currie et al. (2010). The dereddened $(B-V)_{\mathrm{J}, 0 \text {-color was computed as }}$

$$
(B-V)_{\mathrm{J}, 0}=1.362\left(B_{2}-V_{1}\right)_{\mathrm{G}, 0}+0.197
$$

(Meylan \& Hauck 1981) with $\left(B_{2}-V_{1}\right)_{\mathrm{G}, 0}$ the dereddened $\left(B_{2}-V_{1}\right)$-color in the Geneva system. With the interstellar reddening $A_{V}$ and the adopted distance modulus $\mu=5 \log (d)-$ 5 of $11.71 \pm 0.1 \mathrm{mag}$ (see Saesen et al. 2010, Section 7.2), we 

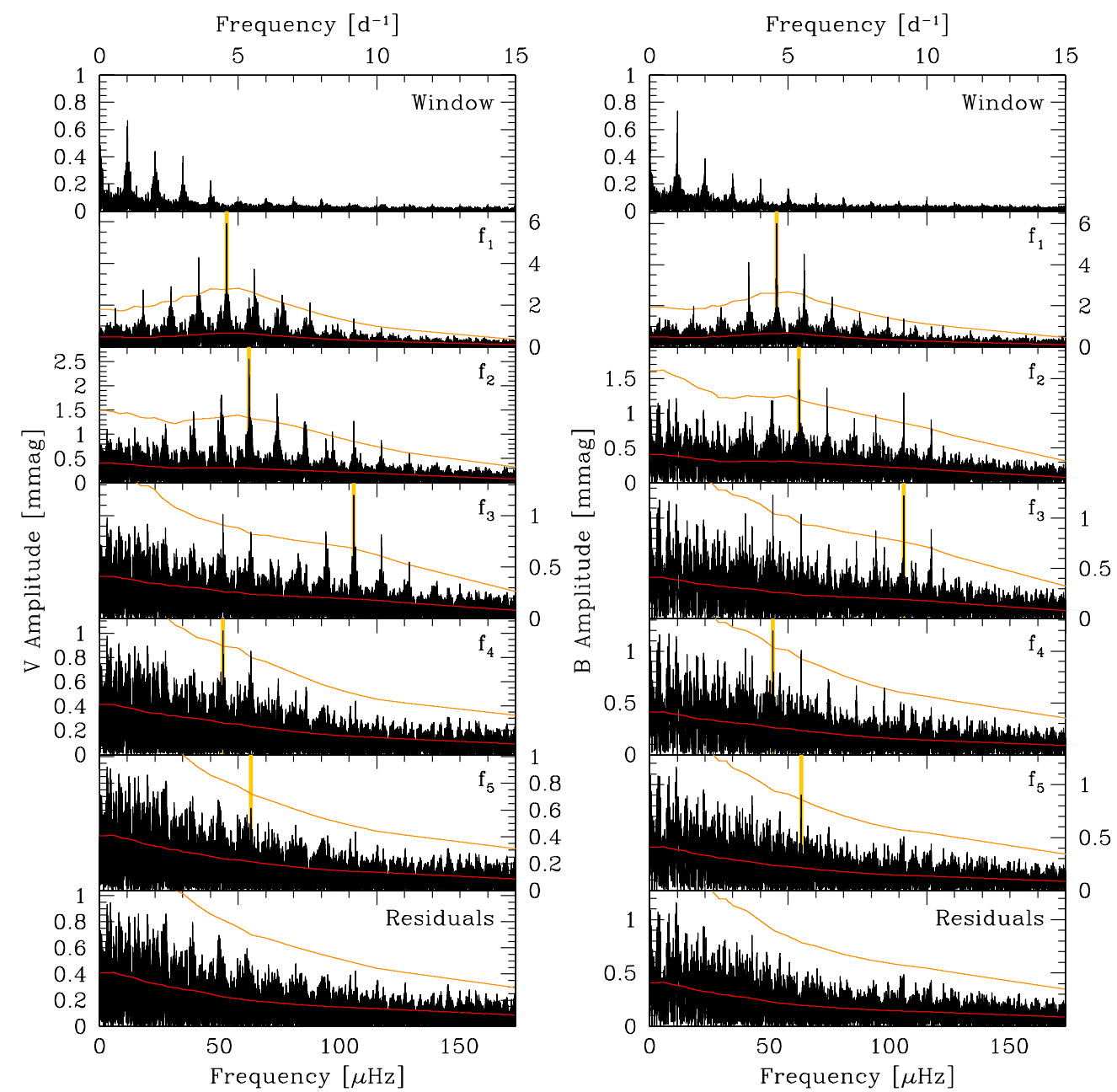

Figure 1. Weighted frequency analysis of Oo 2444. We show the spectral window (top) and amplitude spectra in the different steps of subsequent prewhitening in the $V$ (left) and $B$ (right) filter. The accepted frequencies are marked by a yellow band, the red line corresponds to the noise level, and the orange line to the $4 \mathrm{~S} / \mathrm{N}$ level.

Table 1

The Basic Stellar Parameters $T_{\text {eff }}$ and $\log \left(L / L_{\odot}\right)$ Determined for the Periodic B Stars Based on Our Mean Geneva Colors

\begin{tabular}{|c|c|c|c|c|c|c|c|c|}
\hline Star ID & $\begin{array}{l}T_{\text {eff }} \\
(\mathrm{K})\end{array}$ & $\begin{array}{c}\log \left(L / L_{\odot}\right) \\
(\operatorname{dex})\end{array}$ & Star ID & $\begin{array}{l}T_{\text {eff }} \\
(\mathrm{K})\end{array}$ & $\begin{array}{c}\log \left(L / L_{\odot}\right) \\
(\text { dex })\end{array}$ & Star ID & $\begin{array}{l}T_{\text {eff }} \\
(\mathrm{K})\end{array}$ & $\begin{array}{c}\log \left(L / L_{\odot}\right) \\
\quad(\operatorname{dex})\end{array}$ \\
\hline Oo 1990 & $13,750 \pm 160$ & $2.27 \pm 0.10$ & Oo 2267 & $14,380 \pm 90$ & $2.39 \pm 0.10$ & Oo 2426 & $12,810 \pm 70$ & $2.08 \pm 0.11$ \\
\hline Oo 2006 & $15,470 \pm 120$ & $2.67 \pm 0.09$ & Oo 2285 & $19,670 \pm 220$ & $2.96 \pm 0.08$ & Oo 2429 & $16,560 \pm 130$ & $2.66 \pm 0.09$ \\
\hline Oo 2037 & $14,590 \pm 210$ & $2.51 \pm 0.10$ & Oo 2299 & $30,200 \pm 580$ & $4.75 \pm 0.08$ & Оо 2444 & $22,850 \pm 1180$ & $4.34 \pm 0.09$ \\
\hline Oo 2091 & $18,080 \pm 170$ & $3.30 \pm 0.09$ & Oo 2309 & $15,000 \pm 90$ & $2.60 \pm 0.10$ & Oo 2455 & $17,450 \pm 160$ & $2.85 \pm 0.09$ \\
\hline Oо 2094 & $21,220 \pm 340$ & $3.33 \pm 0.08$ & Oo 2319 & $14,440 \pm 80$ & $2.44 \pm 0.10$ & Oo 2462 & $20,360 \pm 200$ & $3.42 \pm 0.08$ \\
\hline Oo 2110 & $15,710 \pm 110$ & $2.74 \pm 0.09$ & Oo 2323 & $11,770 \pm 50$ & $1.79 \pm 0.11$ & Oo 2482 & $11,140 \pm 50$ & $1.68 \pm 0.11$ \\
\hline Oo 2114 & $21,810 \pm 210$ & $3.67 \pm 0.08$ & Oo 2324 & $12,270 \pm 50$ & $1.96 \pm 0.11$ & Oo 2488 & $25,450 \pm 660$ & $4.22 \pm 0.08$ \\
\hline Oo 2116 & $13,860 \pm 80$ & $2.29 \pm 0.10$ & Oo 2345 & $15,730 \pm 120$ & $2.54 \pm 0.09$ & Oo 2507 & $17,110 \pm 150$ & $2.88 \pm 0.09$ \\
\hline Oo 2139 & $22,830 \pm 230$ & $3.59 \pm 0.08$ & Oo 2349 & $15,580 \pm 120$ & $2.63 \pm 0.09$ & Oo 2515 & $14,710 \pm 100$ & $2.31 \pm 0.10$ \\
\hline Oo 2185 & $17,030 \pm 150$ & $3.48 \pm 0.09$ & Oo 2350 & $14,960 \pm 100$ & $2.39 \pm 0.10$ & Oo 2524 & $12,740 \pm 60$ & $2.06 \pm 0.11$ \\
\hline Oo 2189 & $18,290 \pm 180$ & $3.04 \pm 0.09$ & Oо 2352 & $15,680 \pm 130$ & $2.85 \pm 0.09$ & Oo 2531 & $11,950 \pm 40$ & $1.90 \pm 0.11$ \\
\hline Oo 2191 & $18,970 \pm 170$ & $3.33 \pm 0.09$ & Oo 2370 & $12,280 \pm 60$ & $1.92 \pm 0.11$ & Oo 2562 & $12,810 \pm 70$ & $1.94 \pm 0.11$ \\
\hline Oo 2228 & $11,740 \pm 70$ & $2.08 \pm 0.11$ & Oo 2371 & $24,400 \pm 600$ & $4.49 \pm 0.08$ & Oо 2566 & $30,060 \pm 720$ & $4.25 \pm 0.08$ \\
\hline Oо 2235 & $22,570 \pm 840$ & $4.37 \pm 0.08$ & Oo 2372 & $22,140 \pm 240$ & $3.47 \pm 0.08$ & Oo 2572 & $23,180 \pm 550$ & $4.14 \pm 0.08$ \\
\hline Oo 2242 & $19,790 \pm 420$ & $3.63 \pm 0.08$ & Oo 2377 & $23,730 \pm 410$ & $3.75 \pm 0.08$ & Oo 2579 & $18,790 \pm 160$ & $3.18 \pm 0.09$ \\
\hline Oo 2246 & $24,440 \pm 270$ & $4.22 \pm 0.08$ & Oo 2406 & $13,020 \pm 60$ & $2.07 \pm 0.11$ & Oo 2601 & $21,600 \pm 220$ & $3.85 \pm 0.08$ \\
\hline Oo 2253 & $15,330 \pm 120$ & $2.70 \pm 0.09$ & Oo 2410 & $12,140 \pm 80$ & $1.88 \pm 0.11$ & & & \\
\hline Oo 2262 & $24,230 \pm 600$ & $3.98 \pm 0.08$ & Oo 2414 & $10,680 \pm 60$ & $1.87 \pm 0.11$ & & & \\
\hline
\end{tabular}

Notes. The first column denotes the star number, the second column the effective temperature in kelvin, and the third column the luminosity in dex. The noted uncertainties on the effective temperature are interpolation errors in the grid of atmosphere models as provided by CALIB. The adopted and more realistic errors are taken as $\Delta T_{\text {eff }}=1000 \mathrm{~K}$, except if the interpolation error would exceed this value. Values denoted in italics are found by extrapolation outside the calibration tables and should be used with caution. 
Table 2

Same as Table 1, but for the B Stars for which We Did Not Obtain Absolute Photometry

\begin{tabular}{lccccc}
\hline \hline Star ID & $\begin{array}{c}T_{\text {eff }} \\
(\mathrm{K})\end{array}$ & $\begin{array}{c}\log \left(L / L_{\odot}\right) \\
(\mathrm{dex})\end{array}$ & Star ID & $\begin{array}{c}T_{\text {eff }} \\
(\mathrm{K})\end{array}$ & $\begin{array}{c}\log \left(L / L_{\odot}\right) \\
(\mathrm{dex})\end{array}$ \\
\hline Oo 1898 (Oo 2224) & $14,830 \pm 110$ & $2.23 \pm 0.10$ & Oo 2520 (Oo 2601) & $21,600 \pm 220$ & $3.84 \pm 0.08$ \\
Oo 1973 (Oo 2126) & $9740 \pm 120$ & $1.44 \pm 0.11$ & Oo 2563 (Oo 2566) & $30,060 \pm 720$ & $4.21 \pm 0.08$ \\
Oo 1980 (Oo 2337) & $12,540 \pm 60$ & $2.26 \pm 0.11$ & Oo 2611 (Oo 2507) & $17,110 \pm 150$ & $2.86 \pm 0.09$ \\
Oo 2019 (Oo 2189) & $18,290 \pm 180$ & $3.04 \pm 0.09$ & Oo 2616 (Oo 2406) & $13,020 \pm 60$ & $2.06 \pm 0.11$ \\
Oo 2086 (Oo 2091) & $18,080 \pm 170$ & $3.30 \pm 0.09$ & Oo 2622 (Oo 2053) & $19,640 \pm 250$ & $3.26 \pm 0.08$ \\
Oo 2089 (Oo 2200) & $16,450 \pm 160$ & $2.77 \pm 0.09$ & Oo 2633 (Oo 2053) & $19,640 \pm 250$ & $3.23 \pm 0.08$ \\
Oo 2141 (Oo 2211) & $14,630 \pm 90$ & $2.53 \pm 0.10$ & Oo 2649 (Oo 2262) & $24,230 \pm 600$ & $3.96 \pm 0.08$ \\
Oo 2146 (Oo 2442) & $12,920 \pm 60$ & $2.10 \pm 0.11$ & Oo 2694 (Oo 2444) & $22,850 \pm 1180$ & $4.34 \pm 0.09$ \\
Oo 2151 (Oo 2412) & $9060 \pm 90$ & $2.02 \pm 0.10$ & Oo 2725 (Oo 1990) & $13,750 \pm 160$ & $2.27 \pm 0.10$ \\
Oo 2245 (Oo 2455) & $17,450 \pm 160$ & $2.88 \pm 0.09$ & Oo 2752 (Oo 2110) & $15,710 \pm 110$ & $2.75 \pm 0.09$ \\
Oo 2342 (Oo 2037) & $14,590 \pm 210$ & $2.47 \pm 0.10$ & Oo 2753 (Oo 2501) & $12,590 \pm 50$ & $2.07 \pm 0.11$ \\
Oo 2448 (Oo 2211) & $14,630 \pm 90$ & $2.52 \pm 0.10$ & & & \\
\hline
\end{tabular}

Notes. The stars with most similar values for $V, B-V$, and $V-I$, on which the calibrations are based, are denoted in parentheses after the star identification.

Table 3

The Basic Stellar Parameters $T_{\text {eff }}$ and $\log \left(L / L_{\odot}\right)$ for Stars that Have, Besides Our Estimates, Other Parameter Estimates from Photometric Calibrations or Spectroscopic Literature Values

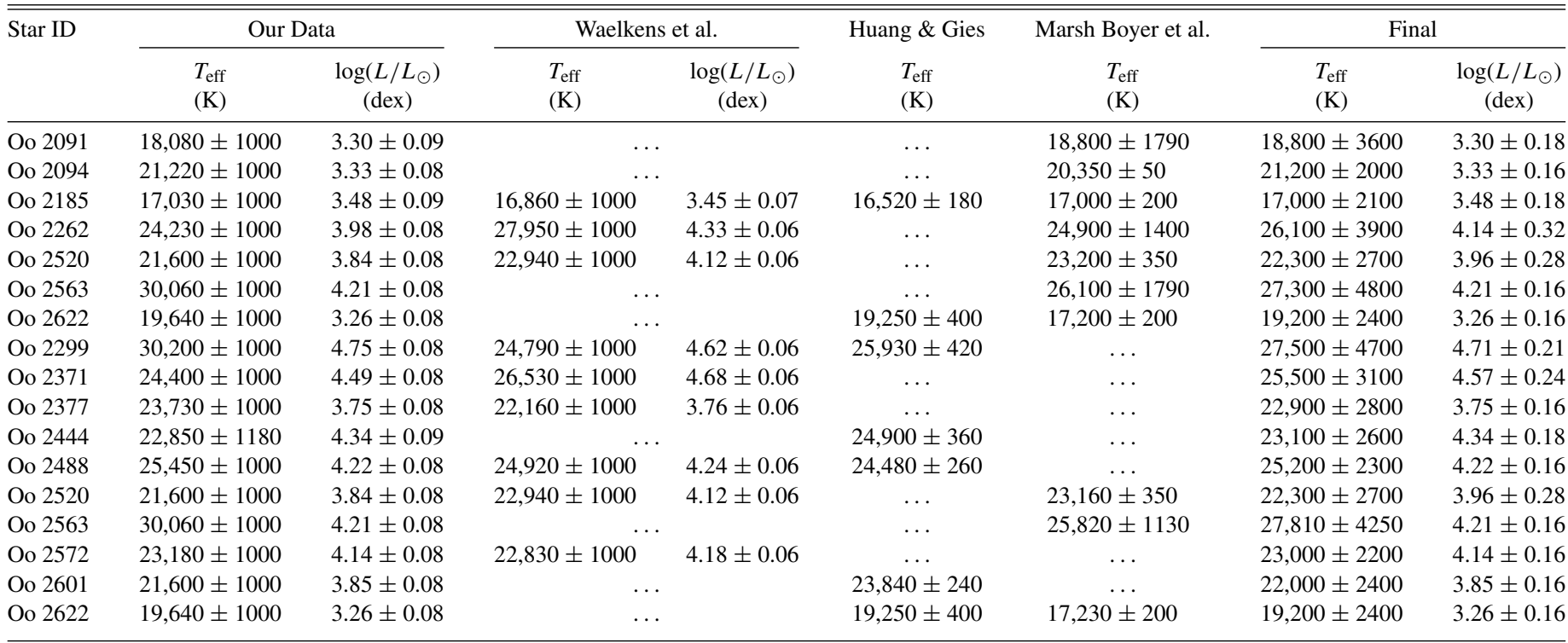

Notes. The first column denotes the star number, the following columns the effective temperature in kelvin and the luminosity in dex for our photometric calibrations, for the photometric calibrations of Waelkens et al. (1990), for the spectroscopy of Huang \& Gies (2006) and Marsh Boyer et al. (2012), and the final adopted values, respectively. For all estimates, the uncertainties noted are $1 \sigma$ error bars; for the final estimates, these are the overall $2 \sigma$ error boxes.

calculated the absolute visual magnitude $M_{V}=V-\mu-A_{V}$. The used distance estimate falls well in the range of recent values listed by Southworth et al. (2004a) and the ones deduced by Currie et al. (2010). Finally, the luminosity was then given by

$$
\log \left(L / L_{\odot}\right)=-0.4\left(M_{\mathrm{bol}}-M_{\mathrm{bol}, \odot}\right)
$$

where we used the bolometric correction of Flower (1996) for $M_{\text {bol }}=M_{V}+\mathrm{BC}$ (see Torres 2010 for the correct coefficients) and $M_{\text {bol, } \odot}=4.73$ mag (Torres 2010).

Table 1 contains the derived values for the luminosity together with their errors, for which uncertainties on the absolute photometry, the reddening, the effective temperature, and the distance were taken into account. We note that, by using the values for the reddening and the distance of the cluster, we implicitly assumed that the stars are cluster members.
For the B stars that were not observed in absolute photometry, we only have the differential photometry to derive their $T_{\text {eff }}$ and $\log \left(L / L_{\odot}\right)$ estimates. For this purpose, we searched for the star with most similar mean $V, B-V$, and $V-I$ measurements and for which we obtained the basic stellar parameters, excluding binary stars. We then made use of the fact that we are working in a cluster and adopted these calibrations for the star of interest. Their effective temperature and luminosity values, together with the star on which we based these estimates, are noted in Table 2.

In the literature, we also find absolute Geneva colors and $T_{\text {eff }}$ values for some B stars in NGC 884. Waelkens et al. (1990) observed the brighter cluster stars with the Geneva photometer attached to the $76 \mathrm{~cm}$ telescope at Jungfraujoch in Switzerland. We used their absolute photometry, their dereddened visual magnitudes, and our distance estimate to determine $T_{\text {eff }}$ and $\log \left(L / L_{\odot}\right)$ values using the same calibrations as described above. The results are noted in Table 3. The study of Slesnick 
et al. (2002) derived the effective temperature for many stars, for some based on photometry, for others based on spectroscopy. However, because they do not provide the uncertainties, we will not take these values into account. The spectroscopic determinations of the effective temperature of Huang \& Gies (2006) and Marsh Boyer et al. (2012) are also noted in Table 3, the latter study being a continuation and extension of the former. In Table 3 we also list our photometric derivations with realistic error bars along with the values from the literature, for comparison. In the last column, we give the final estimates for $T_{\text {eff }}$ and $\log \left(L / L_{\odot}\right)$. These values are calculated as the center of an error box encompassing all the individual $2 \sigma$ error boxes. The noted uncertainty gives the borders of the large overall error box. These final and conservative estimates will be used for the mode identification performed in Section 5 .

It is noteworthy that the projected rotational velocities of cluster members derived by Strom et al. (2005), Huang \& Gies (2006), and Marsh Boyer et al. (2012), which are shown in our Figure 7 below, are typically less than half of the critical velocity. In that case, the above calibrations and those used in the literature, which are all based on the assumption of nondeformed stars, are justified (see, e.g., Figure 1 of Aerts et al. 2004, which shows the value of the local radius, gravity, effective temperature, and luminosity as a function of co-latitude for various values of $\left.v \sin i / v_{\text {crit }}\right)$.

\section{FREQUENCY ANALYSIS}

We performed the frequency analysis with PERIOD04 (Lenz \& Breger 2005). This code was developed to extract individual frequencies of self-driven oscillation modes in the approximation of an infinite mode lifetime, by means of a discrete Fourier transform algorithm. Moreover, it allows us to apply simultaneous multi-frequency sine-wave fitting in the least-squares sense and iterative prewhitening. The advantage is that the code is especially dedicated to the analysis of large astronomical time series containing gaps and that it is able to calculate optimal light-curve fits for multi-periodic signals with the inclusion of harmonic and combination frequencies.

We searched for frequencies in the $V-, B-, I-$, and $U$-filter data. For the $V$ data we used a weighted and non-weighted analysis; for the other filters only a weighted frequency analysis was carried out. For the point weights we used the inverse of the square of the observational uncertainties on each data point, taken from Saesen et al. (2010). For most stars we restricted the computation of the amplitude spectra to the frequency interval $[0-15]$ day $^{-1}$, since the typical B star pulsations range from 0.3 to 1 day $^{-1}$ (for slowly pulsating B stars, SPBs hereafter) and 3 to 12 day $^{-1}$ (for $\beta$ Cep stars) and since it was found in Saesen et al. (2010) that no power occurs beyond 15 day $^{-1}$. We worked in frequency steps of $0.00005 \mathrm{day}^{-1}$. The choice for this step relied on the time base and noise properties of the data, but its precise value is not of importance since the frequency values are optimized in the frequency analysis procedure.

We considered a frequency peak significant when its amplitude exceeds four times the noise level (Breger et al. 1993). We computed this noise level as a running average of the amplitude spectrum. As already argued in Saesen et al. (2010), we considered different intervals over which we evaluated the noise level, according to the frequency value: we used an interval of $1 \mathrm{day}^{-1}$ for $f \in[0-3] \mathrm{day}^{-1}$, of $1.9 \mathrm{day}^{-1}$ for $f \in$ [3-6] day ${ }^{-1}$, of 3.9 day $^{-1}$ for $f \in[6-11] \mathrm{day}^{-1}$, and of $5 \mathrm{day}^{-1}$ for $f \in[11-15] \mathrm{day}^{-1}$. We allow lower significance levels for harmonic and combination frequencies, or if the frequency value occurs in different filters. We then relax the criterion to a signal-to-noise ratio $(\mathrm{S} / \mathrm{N})$ level of 3.6 instead of 4.0, as is justified for B stars (Handler et al. 2004, 2006, 2012). With these different intervals for the noise computation, we are stricter than taking the conventional approach of computing the noise over a 5 day $^{-1}$ range (e.g., Handler et al. 2012), while still being computationally efficient.

We describe here the different steps of our frequency analysis by means of one example star, Oo 2444, which was suspected to be variable with a period of 0.17 days by Krzesiński (1998) and confirmed as $\beta$ Cep star by Pigulski et al. (2007), but no frequencies were reported so far. We started our analysis by calculating the spectral window and the amplitude spectrum of the data (see first two panels of Figure 1 for the weighted analysis of the $V$ and $B$ data). In the amplitude spectrum, one clear peak $f_{1}$ dominates. The peak structure, which is not explained by the spectral window, is a signature of additional frequencies. This first frequency also appears as a significant peak in all other filters.

We prewhitened the data by subtracting a sine wave from the original data. The frequency, amplitude, and phase of this sine wave were optimized in the $V$ filter, since the time span of the light curves is largest in this filter and yields most data points. For the $B, I$, and $U$ light curve, we fixed the frequency value and optimized only the amplitude and phase of the variation.

We then proceeded by searching for frequencies in the residual light curve by again calculating its amplitude periodogram (see third panel of Figure 1). We detect a second signal at frequency $f_{2}$ in $V, B$, and $I$, but not in $U$. This is not surprising given the limited number of measurements in the $U$ filter. We prewhitened the original data, again by optimizing the frequency values of $f_{1}$ and $f_{2}$ simultaneously in the $V$ filter and imposing these frequencies in the multi-frequency fit of $B, I$, and $U$, leaving only the amplitude and phase as free parameters.

The subsequent frequency in the residual light curve $f_{3}$ is equal to $2 f_{1}$ within its uncertainty (see fourth panel of Figure 1). This signal was again significantly present in both the $V, I$ and $B$ data set. We thus removed a three-frequency fit from the data, using the same optimization method as before and fixing the third frequency to the exact harmonic of the first frequency. Hereafter we retain $f_{4}$ (see fifth panel of Figure 1), which is significant in $V$ and $B$, but not in $I$ and $U$.

In the residuals of the four-frequency fit, no significant frequencies are left, except in the $B$ filter, where we retrieve frequency $f_{5}$ (see sixth panel of Figure 1). We verified that including $f_{5}$ in the frequency solution for $V$ and $I$ did not change the fitted amplitude for the frequencies $f_{1}$ to $f_{4}$. Optimizing all frequencies in the $B$ filter did not converge to the same frequency solution as in the $V$ filter within the uncertainty; therefore, we optimize $f_{1}$ to $f_{4}$ in $V$ and $f_{5}$ in $B$. The residuals after prewhitening with the five-frequency fit do not show any significant frequencies anymore (see seventh panel of Figure 1).

An overview of the properties of the final five-frequency fit is given in Table 4. The uncertainties on the frequency, amplitude, and phase values are computed from the error matrix of the least-squares harmonic fitting, as provided by PERIOD04, where we decoupled the frequencies and phases.

Another error estimate for the frequency $f_{i}$ is given by $\Delta f_{i}=\sqrt{6} \sigma / \sqrt{N} \pi T A_{i}$, where $\sigma$ is the standard deviation of the final residuals, $N$ is the number of data points, $T$ is the total time span, and $A_{i}$ is the amplitude of frequency $f_{i}$ (Montgomery \& O'Donoghue 1999). This analytical formula gives an overestimation of the accuracy. A more realistic value 
Table 4

Results of the Multi-frequency Fit to the $U, B, V$, and $I$ Light Curves of All B Stars

\begin{tabular}{|c|c|c|c|c|c|c|c|c|c|c|c|c|c|}
\hline \multirow{2}{*}{$\begin{array}{l}f_{i} \\
\left(\text { day }^{-1}\right)\end{array}$} & \multicolumn{3}{|c|}{$U$} & \multicolumn{3}{|c|}{$B$} & \multicolumn{3}{|c|}{$V$} & \multicolumn{3}{|c|}{$I$} & \multirow[b]{2}{*}{$\ell$} \\
\hline & $\begin{array}{c}A_{i} \\
(\mathrm{mmag})\end{array}$ & $\phi_{i}$ & $\mathrm{~S} / \mathrm{N}$ & $\begin{array}{c}A_{i} \\
(\mathrm{mmag})\end{array}$ & $\phi_{i}$ & $\mathrm{~S} / \mathrm{N}$ & $\begin{array}{c}A_{i} \\
(\mathrm{mmag})\end{array}$ & $\phi_{i}$ & $\mathrm{~S} / \mathrm{N}$ & $\begin{array}{c}A_{i} \\
(\mathrm{mmag})\end{array}$ & $\phi_{i}$ & $\mathrm{~S} / \mathrm{N}$ & \\
\hline \multicolumn{14}{|l|}{ Oo 2094} \\
\hline$f_{1}=0.37596(4)$ & $7(1)$ & $0.49(3)$ & 3.7 & $4.9(5)$ & $0.46(2)$ & 5.0 & $4.5(2)$ & $0.439(9)$ & 4.1 & $4.3(5)$ & $0.46(2)$ & 4.8 & $1,2,4$ \\
\hline$f_{2}=2 f_{1}$ & $2(1)$ & $0.4(1)$ & $\cdots$ & $2.2(5)$ & $0.47(4)$ & 3.9 & $1.3(2)$ & $0.24(3)$ & 3.2 & $1.6(5)$ & $0.37(5)$ & $\cdots$ & $\cdots$ \\
\hline \multicolumn{14}{|l|}{ Oo 2246} \\
\hline$f_{1}=5.429245(7)$ & $6(1)$ & $0.17(3)$ & 5.5 & $6.0(3)$ & $0.169(9)$ & 8.6 & $5.7(1)$ & $0.168(4)$ & 8.9 & $5.1(2)$ & $0.166(7)$ & 7.3 & 2 \\
\hline$f_{2}=5.85616(1)$ & $3(1)$ & $0.01(8)$ & 3.6 & $3.3(3)$ & $0.07(2)$ & 8.7 & $3.2(1)$ & $0.078(7)$ & 8.9 & $3.4(2)$ & $0.07(1)$ & 8.9 & 4 \\
\hline$f_{3}=5.02348(4)$ & $2(1)$ & $0.5(1)$ & 3.2 & $1.0(3)$ & $0.51(5)$ & 4.2 & $0.6(1)$ & $0.51(4)$ & 3.7 & $0.7(2)$ & $0.53(5)$ & 3.4 & 0 \\
\hline \multicolumn{14}{|l|}{ Oo 2444} \\
\hline$f_{1}=4.58161(2)$ & $7(2)$ & $0.63(5)$ & 7.2 & $6.1(3)$ & $0.622(7)$ & 9.5 & $5.9(1)$ & $0.624(4)$ & 8.9 & $4.8(2)$ & $0.624(8)$ & 7.8 & 1 \\
\hline$f_{2}=5.39333(5)$ & $2(2)$ & $0.3(2)$ & $\cdots$ & $1.9(3)$ & $0.30(2)$ & 6.0 & $2.3(1)$ & $0.297(9)$ & 7.9 & $1.8(2)$ & $0.29(2)$ & 5.5 & / \\
\hline$f_{3}=2 f_{1}$ & $1(2)$ & $0.5(2)$ & 3.3 & $1.2(3)$ & $0.59(4)$ & 6.4 & $1.2(1)$ & $0.57(2)$ & 7.1 & $0.9(2)$ & $0.57(4)$ & 5.0 & $\cdots$ \\
\hline$f_{4}=4.4494(1)$ & $1(2)$ & $0.2(4)$ & $\cdots$ & $1.2(3)$ & $0.13(4)$ & 4.7 & $1.0(1)$ & $0.12(2)$ & 4.6 & $0.8(2)$ & $0.15(4)$ & 3.3 & $0, \mathbf{1}, \mathbf{2}, 3,4$ \\
\hline$f_{5}=5.4643(3)$ & $1(2)$ & $0.4(3)$ & $\cdots$ & $1.0(3)$ & $0.44(5)$ & 4.2 & $0.6(1)$ & $0.49(3)$ & 3.4 & $0.8(2)$ & $0.44(5)$ & 3.7 & / \\
\hline
\end{tabular}

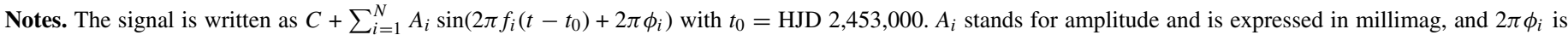

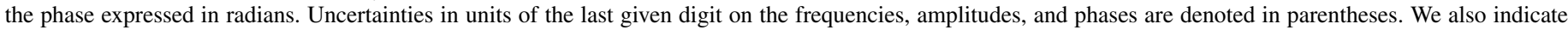

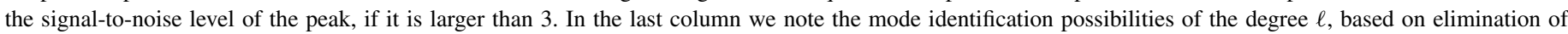

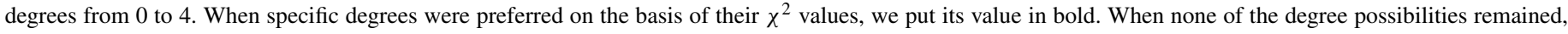

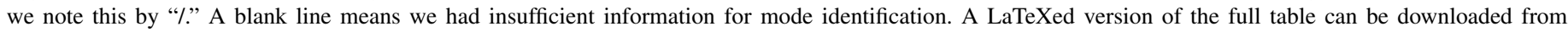
http://arxiv.org/abs/1307.4256 in pdf format.

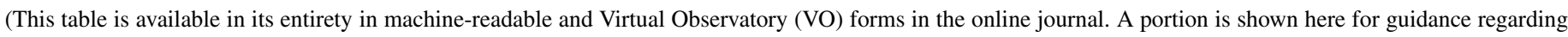
its form and content.)

is obtained when taking the correlations of the data into account, by multiplying the uncertainties by $\sqrt{\mathrm{D}}$, where $\mathrm{D}$ is the number of consecutive data points which are correlated (SchwarzenbergCzerny 1991). For our data set of Oo 2444, this gives errors of $0.6,1,3$, and $5 \times 10^{-5}$ day $^{-1}$ for $f_{1}, f_{2}, f_{4}$, and $f_{5}$, respectively, so that these error estimates deviate less than a factor 6 from the formal least-squares uncertainties listed in Table 4.

Section 6 contains the results for all the other variable B stars, where we followed the same approach.

\section{MODE IDENTIFICATION}

Successful seismic modeling not only involves detecting pulsation frequencies but also relies on the identification of the spherical wavenumbers $(\ell, m)$ of the modes. Unfortunately, unambiguous identifications of detected frequencies are rather scarce for heat-driven pulsators, given that we are not in the asymptotic regime of the frequencies. Our collected photometry of NGC 884 in different colors in principle allows deducing the degree $\ell$ of the various modes of the pulsators. For this purpose we used the well-known method of photometric amplitude ratios, which compares the observational values with the theoretical ones. This technique has already been successfully applied for B-type stars (see, e.g., Heynderickx et al. 1994; Aerts 2000; Handler et al. 2003; De Ridder et al. 2004; De Cat et al. 2007). As can be seen in Table 4, no phase differences within the observational errors between the different filters are detected, so we restrict to the amplitude ratios to determine the degree $\ell$, following the method of Dupret et al. (2003).

\subsection{Grid of Stellar Models and Pulsation Computations}

We made use of an extensive grid of equilibrium models calculated by the evolutionary code CLÉS (Code Liégeois d'Évolution Stellaire; Scuflaire et al. 2008b). The models were computed using OP opacity tables (Seaton 2005) assuming the
Asplund et al. (2005) solar abundance mixture. We used gray atmosphere models. The reader is referred to Briquet et al. (2011) for more details on the adopted input physics. A given model is characterized by five parameters: its mass $M$, its initial hydrogen abundance $X$, its initial metallicity $Z$, its core convective overshoot parameter $\alpha_{\mathrm{ov}}$, and its age. The grid consists of main-sequence models with masses from 2 to $20 M_{\odot}$, with a step of $0.1 M_{\odot}$, four values for the initial hydrogen abundance $X=0.68,0.70,0.72,0.74$, five values for the initial metallicity $Z=0.010,0.012,0.014,0.016,0.018$, and an overshoot parameter $\alpha_{\text {ov }}$ between 0.00 and 0.50 in steps of 0.05 . In total, this grid contains 36,000 evolutionary tracks from the start to the end of the central hydrogen burning phase (ZAMS to TAMS). It consists of over 3 million models in the core-hydrogen burning phase, the hydrogen-shell burning phase being too short to consider in comparison with the main-sequence phase (also known as the Hertzsprung gap).

For each of these models, the theoretical frequency spectrum of low-order low-degree axisymmetric ( $m=0)$ modes was computed using the standard adiabatic code osc (Liège Oscillation Code, Scuflaire et al. 2008a). The age step along one evolutionary track is such that the median of the frequency differences of low-order low-degree $p$ - and $g$-modes for consecutive stellar models (which will be used further in Section 7.2) amounts to $0.07 \mathrm{day}^{-1}$ and $0.03 \mathrm{day}^{-1}$, for models of $7 M_{\odot}$ and of $20 M_{\odot}$, respectively, and reaches a value in between those for all the masses in [7, 20] $M_{\odot}$. This grid was already successfully used for the seismic modeling of various isolated massive pulsators (e.g., Desmet et al. 2009; Briquet et al. 2011; Aerts et al. 2011, for recent applications).

The frequency values for B stars in the adiabatic approximation are close to their non-adiabatic counterparts, the difference being less than typically $0.001 \mathrm{day}^{-1}$ (e.g., Section 4 of Briquet et al. 2007). This reflects the fact that the frequencies of $g$-modes and of low- to moderate-order $p$-modes in B stars are 


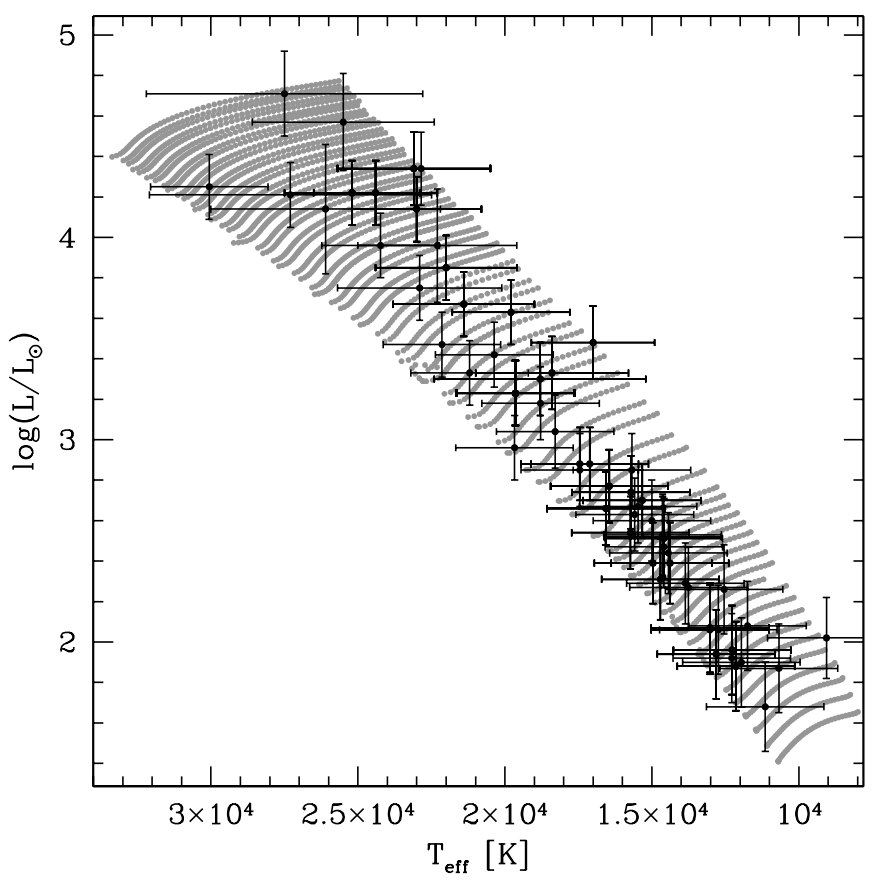

Figure 2. H-R diagram with the model grid for mode identification, as described in the text, denoted in gray. The position of all treated B stars together with their observational $2 \sigma$ error boxes are also shown in black.

determined mostly by the internal layers, where the adiabatic approximation is excellent (see, e.g., Dupret 2002, for a detailed explanation). However, for the purpose of mode identification, we need to compute the non-adiabatic eigenfunctions to predict the theoretical amplitude ratios. This is much more CPU intensive than adiabatic computations and not feasible for the grid of models discussed above. Also, an appropriate sampling of models covering the error boxes in the H-R diagram is largely sufficient, as was demonstrated in previous modern applications of mode identification of B stars (e.g., De Cat et al. 2005, 2007; Handler et al. 2005, 2006). Following these previous studies, we considered a sub-grid of the one discussed above for the mode identification, with masses of $2.1,2.2,2.3, \ldots, 3.9,4.0,4.2$, $4.5,4.7,5.0, \ldots, 15.7$, and $16.0 M_{\odot}$, restricting to $Z=0.010$, $X=0.70$, and $\alpha_{\mathrm{ov}}=0.2$. For all the models in this sub-grid, which is shown in Figure 2, we computed the theoretical eigenfunctions and eigenfrequencies with the non-adiabatic pulsation code MAD (Dupret et al. 2002). Herein, a detailed treatment of the non-adiabatic behavior of the pulsations in the atmosphere is included. We considered all low-order $p$ - and $g$-modes with degrees $\ell$ between 0 and 4 as the chance to observe higher degree modes with photometry is very small due to geometrical cancellation effects. We computed the amplitude ratios associ- ated with the theoretical frequencies with respect to the $V$ filter (see Dupret et al. 2003 for more information) for gray atmosphere models and confronted these with the observed ones in Section 5.2. Before we discuss the concrete applications of mode identification, various remarks are appropriate.

Fixing a value for $Z$ and $\alpha_{\text {ov }}$ is commonly adopted for mode identification of B stars (e.g., Handler et al. 2012, for a recent application). We illustrate the typical theoretical uncertainty on the amplitude ratios for our grid of models in Figure 3. To construct this figure, we selected two typical models for a $\beta$ Cep star with extreme values that have the same position in the H-R diagram. On the one hand, we took a model with $Z=0.01$ and $\alpha_{\mathrm{ov}}=0.5$; on the other hand, we selected a model with $Z=0.02$ and $\alpha_{\mathrm{ov}}=0.0$. For both models and for each degree $\ell$, we selected a frequency with very similar values in each model which fell in the typical range for $\beta$ Cep variations and calculated its theoretical amplitude ratios shown in Figure 3. We see that there is only little difference between the predictions for both models. We tested more cases, varying only $Z$, only $\alpha_{\mathrm{ov}}$, or both together as in Figure 3 . The general conclusion is that the difference in amplitude ratios is usually negligible compared to the observational errors on the observed amplitude ratios we are coping with. This is in agreement with the theoretical uncertainties found by Handler et al. (2005, their Figure 4) based on independent evolution and pulsation codes. Thus, for the data we are dealing with here, the mode identification does not change when changing $\left(Z, \alpha_{\text {ov }}\right)$ because the change in theoretical predictions is smaller than the observed errors.

A second remark concerns the mode instability. In our comparison between observed and theoretical amplitude ratios from the sub-grid, we considered all the computed eigenfrequencies, i.e., as in similar recent applications of mode identification, we did not restrict to modes predicted to be excited (e.g., De Cat et al. 2007; Handler et al. 2012). Our argument is that recent studies have shown there to be problems with the details of excitation computations for various modes in main-sequence OB pulsators, where modes predicted to be stable were detected in the data (e.g., Dziembowski \& Pamyatnykh 2008; Handler et al. 2009; Briquet et al. 2011; Aerts et al. 2011). An extreme case occurred for the O9V pulsator HD 46202, for which none of the detected modes are predicted to be excited. Relying on the instability predictions for mode identifications is thus inappropriate.

Finally, we point out that the pulsational computations done with OSC and MAD ignore rotational effects and only provide axisymmetric modes, just as in Balona et al. (1997), who considered arbitrary equatorial rotation velocities from zero to $200 \mathrm{~km} \mathrm{~s}^{-1}$ to interpret B-type pulsators in young open clusters. Meanwhile, however, the theoretical study by

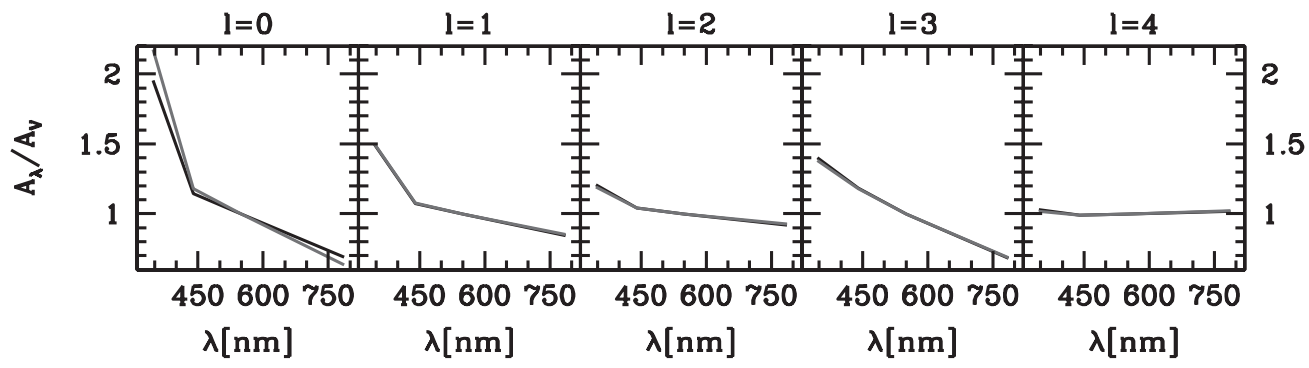

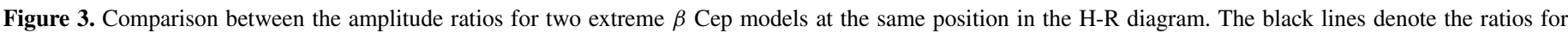

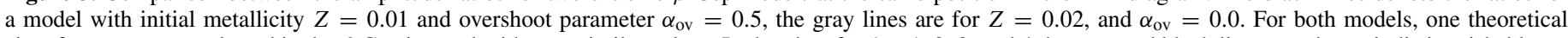
eigenfrequency was selected in the $\beta$ Cep interval with very similar values. In the plots for $\ell=1,2,3$, and 4 the gray and black lines are almost indistinguishable. 

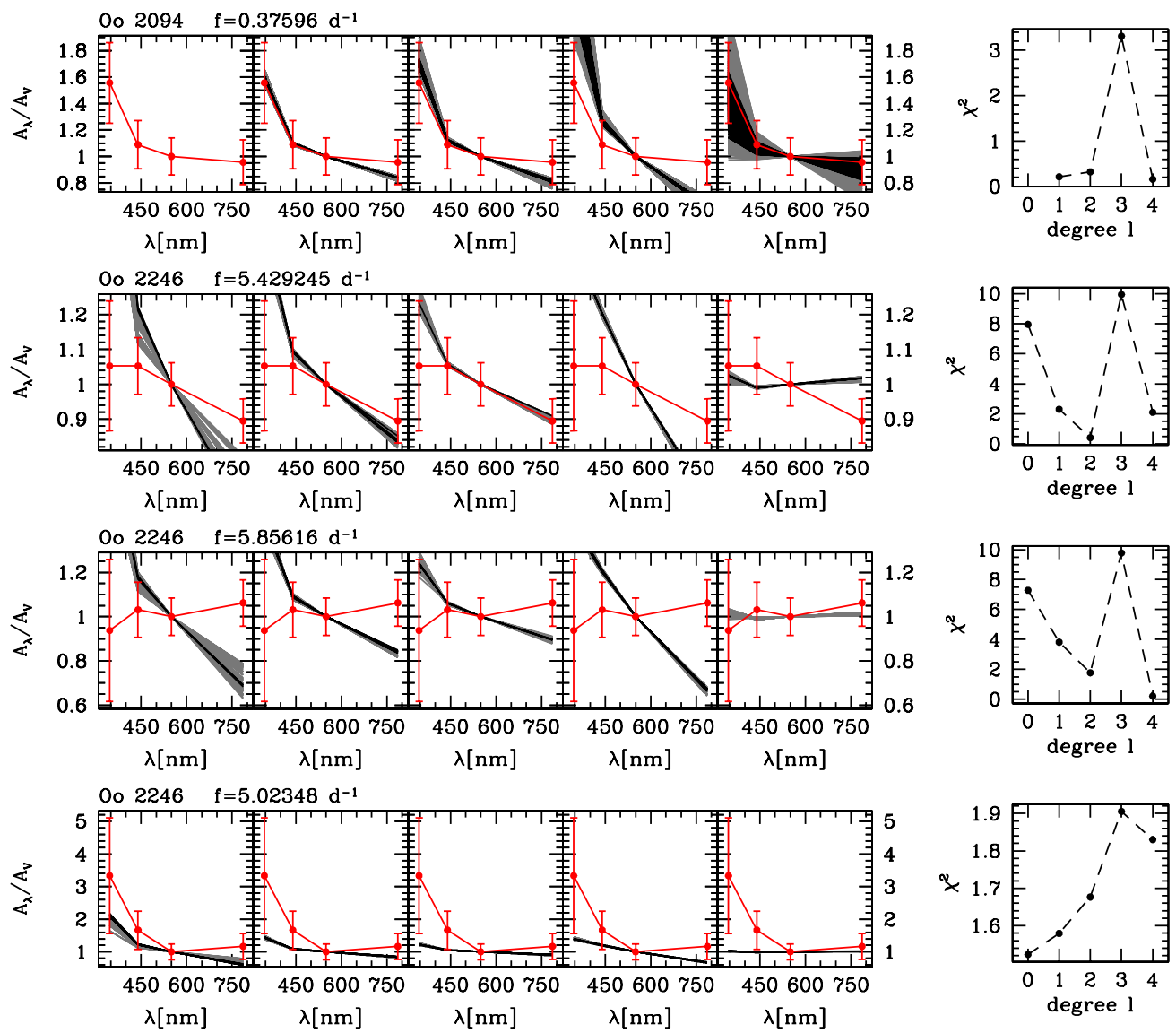

Figure 4. (Left) Amplitude ratios scaled to the $V$ filter for the frequency and the star denoted above the figure. The red filled circles with error bars denote the observed amplitude ratios and their uncertainties, while the gray bands indicate the theoretical predictions for these, running from $\ell=0$ at the left to $\ell=4$ at the right. The black bands denote a subsample of these theoretical models that deviate only $500 \mathrm{~K}$ in effective temperature and 0.05 dex in luminosity from the observed position in the H-R diagram. (Right) Corresponding $\chi^{2}$ value as a function of the degree $\ell$.

(The complete figure set (106 images) is available in the online journal.)

Daszyńska-Daszkiewicz et al. (2002) took into account the effects of rotation on photometric mode identification from amplitude ratios, while considering a typical $\beta$ Cep star with an equatorial rotation velocity of $100 \mathrm{~km} \mathrm{~s}^{-1}$. They pointed out that the effects on the amplitude ratios and on the phase differences can be significant, depending on the mode degree and its coupling with modes of other degrees. Unfortunately, this theoretical study is of no practical use in mode identification when the equatorial rotation velocity and stellar inclination angle are not known, which is the case for the pulsating B stars in NGC 884. On the other hand, we note that Daszyńska-Daszkiewicz et al. (2002) found large rotational effects on the amplitude ratios to be accompanied by large phase differences of the light curves in the different wavebands. We did not find any significant phase differences for the modes detected in our data, which points out that the effects of rotation, if any, are limited as they would otherwise result in measurable phase differences for the amplitudes in the different filters. Thus, as did Balona et al. (1997), we perform the mode identification under the assumption that the rotation can be ignored in the computation of the theoretical amplitude ratios, realizing that this may not be optimal for all the modes in all the considered pulsators.

\subsection{Application to the B Stars in NGC 884}

For each pulsator, models in the sub-grid in Figure 2 that fitted the observed position in the H-R diagram within a $2 \sigma$ error box, as derived in Section 3 and noted in Table 1, Table 2, and the last column of Table 3, were retained. For each model and each degree $\ell$, we selected the eigenfrequencies $f_{\mathrm{T}, i}$ that match the observed frequency $f_{i} \pm 0.2$ day $^{-1}$. We take an uncertainty of $0.2 \mathrm{day}^{-1}$ into account since the theoretical eigenfrequencies assume axisymmetric modes. We cannot compute a more exact value for the rotational splitting due to unknown inclination and rotation rate of the star, in combination with the unknown azimuthal number $m$. Our approach is stricter than in the previous applications mentioned above (De Cat et al. 2005, 2007; Handler et al. 2006, 2005), where the closest frequency in their used model grid, which was in addition much less dense than ours, was selected, irrespective of the difference with the theoretical frequency value. We have therefore followed a particularly conservative approach in assigning mode identifications to our detected frequencies.

We confronted the theoretical amplitude ratios retained in this way with those observed. We illustrate the results of the mode identification by two examples. The outcome for all B stars treated in this section can be found in Table 4 and Figure 4. Our results rely on the selected sub-grid combined with the elimination of certain degrees $\ell$ for the $2 \sigma$ error box in the H-R diagram of each star as described in the previous paragraph.

The results for Oo 2094 are based on the observed amplitude values presented in Table 4 . The mode identification plots with the comparison between the theoretical and observational amplitude ratios are shown in the top left panel of Figure 4. First of all, we eliminate the possibility of degree $\ell=0$. None of the 
models in the grid that fall in the $2 \sigma$ error box of Oo 2094 in the H-R diagram have $(\ell=0)$-modes with frequencies close enough $\left( \pm 0.2 \mathrm{day}^{-1}\right)$ to $f_{1}=0.37596 \mathrm{day}^{-1}$. This is not at all surprising since the frequency value of $f_{1}$ already indicated that we are probably dealing with a $g$-mode. We can also eliminate $\ell=3$, because the theoretical amplitude ratios for the $I$ filter never match the observed one within the uncertainties. We retain $\ell=1,2$, and 4 , but remark that the observational amplitude ratios follow best the theoretical band for $\ell=4$.

The mode identification for the three frequencies detected in Oo 2246, $f_{1}=5.429245 \mathrm{day}^{-1}, f_{2}=5.85616 \mathrm{day}^{-1}$, and $f_{3}=5.02348 \mathrm{day}^{-1}$, is shown in the lower three left panels of Figure 4. The observed amplitude ratios are only compatible with the theoretical models with $\ell=2$ for $f_{1}, \ell=4$ for $f_{2}$, and $\ell=0$ for $f_{3}$, although the dependence of the observed ratios on wavelength does not always resemble the theoretical one. We also note that, even though we are just on the limit of eliminating the possibility of $\ell=1$ for $f_{3}$, there is not much reason to prefer $\ell=0$ to $\ell=1$.

We also identified the mode degree from an alternative approach, as in, e.g., De Ridder et al. (2004) and Handler et al. (2006), by calculating a $\chi^{2}$ value for every frequency and every degree $\ell$, as

$$
\chi^{2}(\ell)=\frac{1}{\sqrt{N-1}} \sum_{j=1}^{N}\left(\frac{A_{j, \mathrm{th}} / A_{V, \mathrm{th}}-A_{j, \mathrm{obs}} / A_{V, \mathrm{obs}}}{\sigma_{j, \mathrm{obs}}}\right)^{2},
$$

with $N$ the total number of filters available for the star and $\sigma_{j, \text { obs }}$ the error on the observed amplitude ratio for filter $j$ (see also Aerts 2000; Aerts et al. 2010). The same models were selected as described above, and the $\chi^{2}$ value for the best-fitting model was retained for every degree, i.e., the mode with minimal $\chi^{2}$ is preferred. The outcome for the stars described above is shown in the right panels of Figure 4, and the result is in agreement with the one of the left panels of these figures. The $\chi^{2}$ value indeed expresses the difference of the observed mean amplitude ratio to the one predicted from the closest model. There is no statistically founded acceptance criterion for such $\chi^{2}$ values. However, we note that in the examples above, the elimination process relying on the left panels often retains only models with $\chi^{2}<1$. We only use this $\chi^{2}$ value to prefer specific degrees $\ell$ when a clear minimum is visible, as noted in Table 4 by putting these $\ell$ values in bold. We remark that we always included the amplitudes in the $U$ filter, when available, because, although their error is often larger than for the other filters, the calculated $\chi^{2}$ value accounts for this.

The results for all the mode identification attempts are represented in Figure 4. For one star, Oo 2371, which has one frequency, we omitted the mode identification since it is an ellipsoidal binary. We securely identified the degree for 12 of the 114 detected frequencies: 9 of the 64 investigated stars have at least one mode degree identified. The stars and frequencies for which we only retain one possibility for the degree $\ell$ of the modes are the following: $f_{1}=0.32811 \mathrm{day}^{-1}$ in Oo 2191 has $\ell=4 ; f_{2}=3.12734$ day $^{-1}$ in Oo 2242 has $\ell=2 ; f_{1}=5.429245 \mathrm{day}^{-1}, f_{2}=5.85616 \mathrm{day}^{-1}$, and $f_{3}=5.02348 \mathrm{day}^{-1}$ in Oo 2246 have $\ell=2$, 4, and 0 , respectively; $f_{1}=3.07229 \mathrm{day}^{-1}$ in Oo 2262 has $\ell=4$; $f_{1}=3.145150 \mathrm{day}^{-1}$ in Oo 2299 has $\ell=2 ; f_{1}=0.76570 \mathrm{day}^{-1}$ in Oo 2372 has $\ell=4 ; f_{1}=4.58161$ day $^{-1}$ in Oo 2444 has $\ell=1 ; f_{1}=6.16805 \mathrm{day}^{-1}$ in Oo 2488 has $\ell=2$; and $f_{1}=4.41463 \mathrm{day}^{-1}$ and $f_{2}=4.76330 \mathrm{day}^{-1}$ in Oo 2572 have $\ell=2$ and 1 , respectively. The identified modes will turn out to be powerful input for the derivation of cluster properties as we show below. Nevertheless, the number of unambiguous mode identifications is low, in view of the immense observational effort put into the observing campaign. The lack of good photometric capabilities based on ultraviolet filters led to too uncertain amplitudes at blue wavelengths. This propagated to too high uncertainties for the amplitude ratios to be of sufficiently strong diagnostic value to identify the majority of the detected modes. Future campaigns should take this into account.

\section{RESULTS FOR INDIVIDUAL STARS}

In this section, we describe the results of the weighted analysis of the $V, B, I$, and $U$ light curves for individual stars and the mode identification of the detected frequencies. For figures of the light curves, the phase diagram with the dominant frequency, and the periodograms of the treated B stars, we refer to the electronic Appendix A of Saesen et al. (2010). A summary of the pulsational properties of all these stars, such as frequencies, amplitudes, phases, and degrees $\ell$ of the modes, is noted in Table 4.

The manually performed frequency analysis of all these stars was done similarly as described in Section 4 for Oo 2444. In the following, the results of the non-weighted analysis of the $V$ light curves are comparable to the weighted analysis, except if we state otherwise. When the data in a filter were too limited, or the harmonic fit was too bad, we did not note the results of the frequency fit for that filter. Some stars need some additional remarks, which are noted below, sorted on the star number.

The results of the mode identification were obtained in the same way as described in Section 5 for Oo 2094 and Oo 2246. We did not perform a mode identification for harmonic and combination frequencies, since the probability that they are independent frequencies is very small, as we have checked in our extensive grid of frequencies based on theoretical models, e.g., the probability for an independent harmonic frequency is about $2 \times 10^{-4}$. In Table 4, we eliminated degree possibilities when no models were retained for the star and frequency of interest in the case of $\ell=0$ or when no theoretical amplitude ratios matched the observed ones within the uncertainties for the models selected within the $2 \sigma$ error box in the H-R diagram. We also evaluated the calculated $\chi^{2}$ value for the remaining degrees. When a specific degree was preferred based on its $\chi^{2}$ value, we stated so in Table 4 by putting its $\ell$ value in bold. Whenever no possibilities for $\ell=0$ to $\ell=4$ are left, it might be caused by $\ell>4$, as spectroscopic studies show that highdegree modes occur in moderately rotating pulsating B stars (e.g., Aerts et al. 1998; Telting \& Schrijvers 1998; Telting et al. 2001; Ausseloos et al. 2006). It could also be that the moderate rotation affects the observables too much such that the mode identification is erroneous, as pointed out by Handler et al. (2012), or that we are not dealing with a pulsation frequency. The mode identification figures for all the stars are shown in Figure 4. The results of the mode identification in Table 4 should be regarded in combination with these figures, in order to interpret the magnitude of discrepancy between the models and observations.

Oo 1898. After optimization of the light curves and a detailed frequency analysis, no significant frequency peaks could be found any more in star Oo 1898 . We only retain a candidate frequency $f=3.648$ day $^{-1}$ with an $\mathrm{S} / \mathrm{N}$ of 3.9 in the $V$ data.

Oo 1973. After optimization of the light curves and a detailed frequency analysis, no significant frequency peaks could be found any more in star Oo 1973. 
Oo 2086. For star Oo 2086 we find frequency values around 25 and 29 day $^{-1}$, which are not at all expected for B-type pulsators. Based on its observed frequencies, this star is a $\delta$ Sct variable and thus not a cluster member, as already pointed out in Saesen et al. (2010).

Oo 2089. The analysis of Oo 2089 revealed only significant frequencies in the $V$ filter. The frequency value of $f_{2}$ is rather close to $1.003 \mathrm{day}^{-1}$ and so we have to be cautious since it may not be an intrinsic frequency.

Oo 2091. After prewhitening with $f_{1}$ and $f_{2}$, we found an additional significant frequency $f=1.0437 \mathrm{day}^{-1}$ in the weighted and non-weighted analysis of the $V$ residuals. To check whether this is an intrinsic frequency or not, we analyzed the data of Białków (Poland) and Xinglong (China) Observatory separately. Frequency $f=1.0437 \mathrm{day}^{-1}$ only shows up in the Białków data and is totally absent in the Xinglong data. Therefore, we do not accept this frequency. Prewhitening with $f$ did not reveal any other significant frequencies, so we stopped the frequency search at this point.

Oo 2094. After removing a sine fit with the main frequency $f_{1}$ and its harmonic $f_{2}=2 f_{1}$, we obtained the candidate frequency $f=0.479$ day $^{-1}$. This frequency is the highest peak in both the $B$ and $V$ periodograms of the residuals, but its amplitude does not exceed the needed $\mathrm{S} / \mathrm{N}$ to accept it formally, i.e., it has an $\mathrm{S} / \mathrm{N}$ of 3.7 in $V$ and of 3.4 in $B$.

Oo 2110. The two significant frequencies we accepted for Oo 2110 are $f_{1}$ and $f_{2}$. After prewhitening, a third significant frequency, $f=3.0231 \mathrm{day}^{-1}$, showed up in the weighted and non-weighted analysis of the $V$ data. This frequency was not present in the other filters. A separate analysis of the $V$ data of different observatories showed $f$ in the Białków but not in the Xinglong data. Therefore, we did not accept this third frequency as intrinsic for the time being.

Oo 2116 . In Oo 2116 we can only detect one frequency $f_{1}$. In the $I$ data, the alias peak $f_{1}^{\prime}=4.21$ day $^{-1}$ is higher, whereas in $V$ and $B$, the peak at $f_{1}$ itself is highest. Since the $V$ data are less suffering from aliasing, according to the spectral windows, and since in both $V$ and $B$ data $f_{1}$ is highest, we believe this is the right peak.

Oo 2139. We cannot identify any significant peaks in Oo 2139 , but the Fourier spectra of the data in the different filters are dominated by peaks in the range between 0.4 day $^{-1}$ and 0.6 day $^{-1}$.

Oo 2146. Only in the $V$ data of Oo 2146 can a significant frequency be detected: $f=4.013$ day $^{-1}$ with an $\mathrm{S} / \mathrm{N}$ of 4.2. However, its value is equal to four times $1.0027 \mathrm{day}^{-1}$ within its error bars. Since the data for Oo 2146 mainly come from one observatory, Xinglong, we cannot conduct a further analysis to decide whether this frequency could be intrinsic to the star or not. Therefore, we do not include it in a harmonic fit.

Oo 2185 . For this star, no frequency could be accepted in the weighted analysis. Therefore, the parameters of the frequency fit noted in Table 4 are the results of a non-weighted analysis. In the weighted analysis, much importance is given to the precise Białków data, which are spread over the three observing seasons, whereas the Xinglong data, spread over only four months, become important in the non-weighted analysis, due to their overwhelming number. This may be the reason why the nonweighted frequency search gives better results than the weighted one, since it is possible that the frequencies of Oo 2185 are not stable over time, due to its Be nature.

Oo 2189. After the time series optimization, we do not find any significant frequencies any more in Oo 2189, except $f=0.10411 \mathrm{day}^{-1}$ that just reaches an $\mathrm{S} / \mathrm{N}$ of 4 in a nonweighted analysis of the $V$ data. This frequency, however, does not at all show up in a separate analysis of the Białków and Xinglong data, and therefore we do not trust it.

Oo 2191. Oo 2191 was announced as a candidate SPB star by Saesen et al. (2009), and the derivation of the two frequencies $f_{1}$ and $f_{2}$ confirms this pulsational behavior.

Oo 2228. The results from the weighted and non-weighted frequency search for Oo 2228 are not the same. The first frequency in the weighted $V$ analysis is $f_{1}$ with an $\mathrm{S} / \mathrm{N}$ of 4.5 , and it is also marginally present in the $I$ data. In the non-weighted $V$ data we first retrieved $f=0.959$ day $^{-1}$; prewhitening for this frequency gives $f=2.778 \mathrm{day}^{-1}$ or $f_{1}$. It is impossible to distinguish between these two values, so we have to be aware that we may not have taken the correct frequency peak. Prewhitening the weighted and non-weighted data set with $f_{1}$ gave no more significant frequencies that differ enough from a multiple of $1 \mathrm{day}^{-1}$ and that are similar in both data sets. Therefore, we only accept $f_{1}$.

Oo 2235. After a carefully executed analysis of Oo 2235, we cannot find any significant frequencies in the different filters, although the star surely is variable.

Oo 2242. Be star Oo 2242 was discovered to be a variable star by Krzesiński \& Pigulski (1997). Their data showed variations on a timescale typical for $\lambda$ Eri variables. Strong aliasing prevented them from giving the correct frequency peak.

With our data set, we were able to determine a first frequency peak at $f_{1}$. The non-weighted $V$ analysis led to a second significant frequency $f_{2}$. Its value is very close to $2 f_{1}$. However, when we fixed the second frequency as harmonic $2 f_{1}$ in the sine fit, the amplitude of $2 f_{1}$ is much lower than the one for $f_{2}$ in the periodogram, and after prewhitening, we again obtained $f_{2}$. Moreover, since the frequency difference between $2 f_{1}$ and $f_{2}$ is nearly $4 \mathrm{yr}$ aliases, we also fitted $\left(f_{1}-2 \mathrm{yr}^{-1}\right)$ and its first harmonic $\left(2 f_{1}-4 \mathrm{yr}^{-1}\right)$ to the data. This time, the amplitude of $\left(f_{1}-2 \mathrm{yr}^{-1}\right)$ was much lower than the one for $f_{1}$ and the amplitude of the harmonic $\left(2 f_{1}-4 \mathrm{yr}^{-1}\right)$ corresponded to the one of $f_{2}$. Prewhitening gave again an additional frequency $f_{1}$ with an amplitude as expected. This whole analysis points to $f_{2}$ being an independent additional frequency.

We also retrieved these two frequencies when combining our data set with the measurements of Krzesiński \& Pigulski (1997). Since the time span of the data is much longer in this way, the frequency value can be deduced much more accurately. Therefore, we adopted the non-weighted results of the combined data sets in Table 4.

Oo 2245. The periodograms of Oo 2245 in the different filters first showed many long periods that were due to different zero points in the three observing seasons. After adjusting them, the low frequencies were absent in the $V$ and $U$ periodogram, but for the $I$ and $B$ data we needed to fit one low frequency to get to the intrinsic frequencies. After prewhitening with $f_{1}$ and $f_{2}$, only peaks at multiples of $1 \mathrm{day}^{-1}$ appeared, and we stopped the frequency analysis.

Oo 2246. Krzesiński \& Pigulski (1997) discovered Oo 2246 as one of the first two $\beta$ Cep stars in NGC 884. They could disentangle two frequencies in their data that correspond with our $f_{1}$ and $f_{2}$. After prewhitening, we detected a third frequency $f_{3}$ in the $B$ data. We optimized all three frequencies in the $B$ filter, after verifying that the frequency values of $f_{1}$ and $f_{2}$ are the same as the optimized $V$ values within their error bars. Combining our light curve with the one from Krzesiński \& Pigulski (1997) reproduced the same frequencies. Due to the longer time span, 
the results shown in Table 4 are thus obtained in the joined data sets.

Oo 2253. Oo 2253 was reported as an SPB candidate by Saesen et al. (2009), and they extracted three significant frequencies in the data of Białków and Xinglong Observatory. We confirm here their first two frequencies, denoted as $f_{1}$ and $f_{2}$ in Table 4, but we do not detect their third frequency in our merged data set.

Oo 2267. After prewhitening for $f_{1}$ and $f_{2}$, we found a candidate frequency $f=4.546 \mathrm{day}^{-1}$ in the $I$ residuals. This frequency has an $\mathrm{S} / \mathrm{N}$ of 3.3 in $B, 3.4$ in $V$, and 3.7 in $I$, which is not high enough to accept it. Moreover, due to strong aliasing, we cannot be sure to have picked out the correct frequency peak.

Oo 2299. Oo 2299 was discovered as a candidate variable star on a timescale of $6 \mathrm{hr}$ by Percy (1972) and was identified as second $\beta$ Cep star in NGC 884 by Krzesiński \& Pigulski (1997). They report a single periodicity at $f_{1}$. With our data set we cannot detect any other frequencies within the detection threshold either. The results noted in Table 4 originate from the combined data set.

Oo 2319. Two significant intrinsic frequencies, $f_{1}$ and its harmonic $f_{2}=2 f_{1}$, were derived for Oo 2319. Prewhitening gave alias structures of low frequencies, which we did not accept. We searched further, but no other significant peaks emerged.

The phases of $f_{1}$ and $f_{2}$ are the same in the different filters. The amplitude of $f_{1}$ in $U$ seems largest, and the one in $I$ smallest, which is a typical signature for pulsations in B stars. However, they do not differ within their error bars; therefore, we cannot formally exclude the possibility that this star is an ellipsoidal binary.

Oo 2324. Prewhitening Oo 2324 with $f_{1}$ and $f_{2}$ gives a nice peak at $f_{3}$ with an $\mathrm{S} / \mathrm{N}=5.6$ in the weighted analysis of the $V$ residuals. In the other filters, aliasing and higher noise levels hamper the detection of other frequency peaks. The frequency value of $f_{3}$ is close to 3 times $1.0027 \mathrm{day}^{-1}$, so we should be careful with it. However, when analyzing $V$ data of Białków and Xinglong Observatory separately, both sites indicate $f_{3}$ as a significant frequency. Therefore, we are inclined to accept it as an intrinsic frequency. Further prewhitening no longer gave significant frequency peaks.

Oo 2345. The first frequency found in the data of Oo 2345 is very close to 2 day $^{-1}$. We retrieved $f_{1}$ in all filters, and also in the analysis of some observatories separately. A phase diagram folded with this frequency, as shown in Figure A.37 of Saesen et al. (2010), displays a clear sinusoidal variation. We are thus inclined to accept $f_{1}$ as intrinsic frequency of Oo 2345. Removing a sine fit with $f_{1}$ reveals another frequency $f_{2}$, which is present in the $B, V$, and $I$ residuals. Hereafter no more frequency peaks were accepted.

Oo 2371. Oo 2371 is an ellipsoidal binary with orbital period around 5.2 days. The binary star was first discovered as a candidate by Krzesiński \& Pigulski (1997) and later confirmed in spectroscopic data by Malchenko (2007). Our deduced frequency $f_{1}$ is, as expected, twice the orbital frequency, and the amplitudes in the different filters indeed do not follow the typical relation for B-type pulsations. We also note that a frequency analysis in the different observing seasons showed evidence for a changing amplitude over time, pointing to an interacting binary due to a close orbit (Malchenko 2007). In Table 4 we note the frequency as found in the combined data set. We did not execute a mode identification for this star, since we are not dealing with pulsations.
Oo 2406. Frequency $f_{1}$ is significant in the $V$ and $I$ data of Oo 2406. Prewhitening shows the presence of a second frequency peak, in both the $V$ and $I$ residuals, but with a different alias frequency. $I$ data suffer more from aliasing than $V$ data, and so the difference between both peaks is significantly less than for the $V$ filter. Therefore, we adopt $f_{2}$ given by the $V$ residuals as the correct frequency peak.

Oo 2426. After optimization of the light curves in the different filters and a detailed frequency analysis, we do not recover any significant frequencies for star Oo 2426.

Oo 2429. For Oo 2429, the results noted in Table 4 were optimized in the $B$ filter, since this filter led to more significant frequencies. Moreover, optimizing $f_{1}$ in the $B$ filter resulted in the same frequency values within the error as optimizing in $V$.

Oo 2448. The results obtained for Oo 2448 should be treated with caution: the periodograms suffered from severe aliasing. We adopted the frequencies coming from the $B$ periodograms, since its alias frequency was lower than for the $V$ data, as in the spectral window. The highest peaks in $V$ are $f_{1}^{\prime}=0.497$ day $^{-1}$ and $f_{2}^{\prime}=2.430$ day $^{-1}$ with an $\mathrm{S} / \mathrm{N}$ of 4.0 and 4.8 , respectively.

Oo 2488. Oo 2488 was for the first time announced as a variable star with $\beta$ Cep-like oscillations by Pigulski et al. (2007) in a preliminary report on the multi-site campaign on NGC 884. Saesen et al. (2008) derived two significant frequencies, $f_{1}$ and $f_{2}$, in a frequency analysis on the single-site data of Białków Observatory. An analysis of the bi-site data of Białków and Xinglong Observatory by Saesen et al. (2009) led to the same two frequencies. An analysis of the total data set, as carried out here, confirms $f_{1}$ and $f_{2}$ and even permits the detection of a third frequency $f_{3}$.

Oo 2462. As for star Oo 2185, no frequencies could be accepted in a weighted analysis, so that the results in Table 4 come from a non-weighted frequency analysis.

Oo 2562. After prewhitening with significant frequencies $f_{1}$ and $f_{2}$, a third frequency $f_{3}$ is retrieved in the $I$ data with an $\mathrm{S} / \mathrm{N}$ of 4.6. Inclusion of this frequency and optimization in the $I$ filter do not change the harmonic fit for the two first frequencies in any of the filters. Given the large errors, the parameters of the harmonic fit for this third frequency as noted in Table 4 cannot be trusted.

Oo 2566. Pigulski et al. (2007) and Saesen et al. (2008) already mentioned Oo 2566 as a candidate $\beta$ Cep star based on (a part of) the first-season Białków data. A frequency analysis on the whole campaign data set is suffering from long-term variations caused by the Be character of the star. Frequency $f_{1}$, however, recurs in the $V, B$, and $U$ data after removing some arbitrary low frequencies, but is only significant in the $V$ data. Since the chaotic behavior of this Be star hampers our time series analysis, the results in Table 4 should be taken with caution.

Oo 2572. Oo 2572 was discovered and analyzed in the same preliminary reports on the multi-site campaign as for Oo 2488. Saesen et al. (2009) remarked that the analysis of the bi-site data already was important in order to pinpoint the correct frequency peak, in comparison with the single-site data. They derived two frequencies, $f_{1}$ and $f_{2}$, and reported that there is still power present in the residuals, but that no other frequency value could be determined.

We confirm these two frequencies in the frequency search in the whole campaign data set and retrieve a third frequency peak $f_{3}$. After prewhitening with $f_{1}, f_{2}$, and $f_{3}$, no other frequencies can be accepted for Oo 2572. Only in the non-weighted analysis of the $V$ data do we find evidence for a candidate frequency $f=4.389$ day $^{-1}$ with an S/N level of 3.9. 

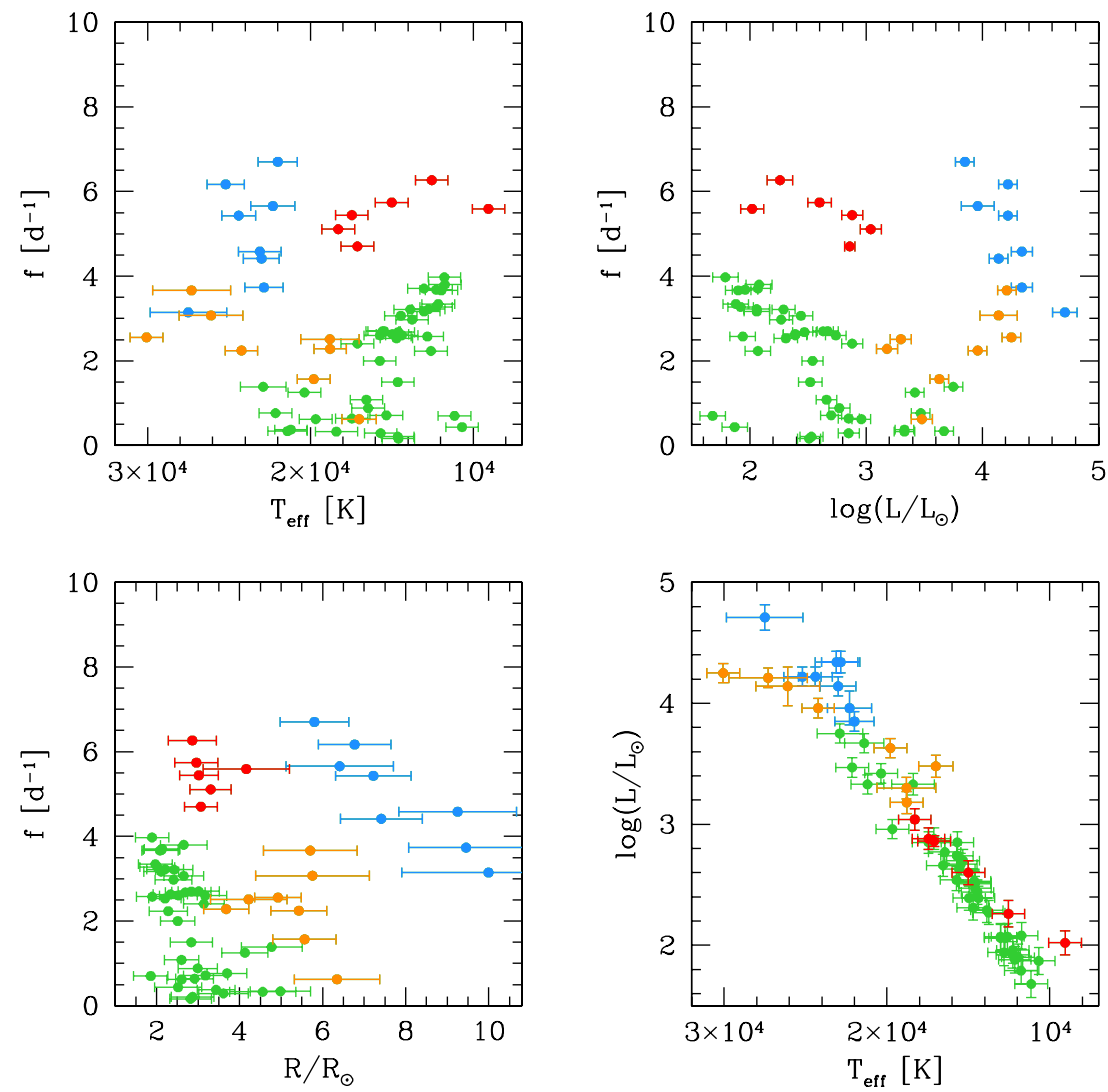

Figure 5. Dominant frequencies for all B stars with respect to the basic stellar parameters $T_{\text {eff }}, \log \left(L / L_{\odot}\right)$, and $R$, with their $1 \sigma$ error. The error on the frequency is smaller than the used symbol. Different colors are attributed to different apparent groups in the $(R, f)$-diagram (see Section 7.1). The bottom right figure shows all these stars in the H-R diagram.

Oo 2579. The results noted in Table 4 for the $I$ data do not include the ones coming from Białków Observatory, since they suffered severely from long-term effects hampering the detection of $f_{1}$. For the other filters, Białków data could be included.

Oo 2616. After prewhitening the $B$ data of Oo 2616 with $f_{1}$, a second frequency $f_{2}$ is found on the limit of acceptance, i.e., with an $\mathrm{S} / \mathrm{N}$ level of 4.0. This frequency was also visible in the first periodogram of the $V$ and $I$ data, and therefore we accept it and include it in the harmonic fit. The parameters of the harmonic fit were optimized in the $B$ filter, but we have no high confidence in them due to their high error bars.

Oo 2622 . Oo 2622 is definitely a variable star, as clearly seen in the $V, B$, and $I$ light curves. However, residual trends hamper a clear and coherent frequency determination in the different filters. Therefore, we do not attempt a harmonic fit for this star.

Oo 2649. We can only accept one frequency in the Be star Oo 2649. However, the phase diagram folded with this frequency (see Figure A.59 in Saesen et al. 2010) clearly shows additional variations. A possible candidate frequency is $f=2.264 \mathrm{day}^{-1}$, which is clearly the highest peak in the $V$ periodogram of the residuals, but its amplitude does not fulfill the $\mathrm{S} / \mathrm{N}$ criteria to accept it.

Oo 2694. For Oo 2694 only one frequency, $f_{1}$, can be accepted, but surely more variation is still hidden in the residuals. The residuals in the different filters pointed toward a different frequency peak; the one in the $V$ data is most significant, at $f=1.502 \mathrm{day}^{-1}$ or one of its aliases, with an $\mathrm{S} / \mathrm{N}=$ 3.8. We cannot accept this frequency, but prewhitening with it even reveals more candidate frequencies. However, we cannot retrieve a trustable value for them, so we stopped the frequency search at this point.

Oo 2752. No significant frequencies can be found in Oo 2752 after optimization of the light curves. We only retain one candidate frequency $f=1.811 \mathrm{day}^{-1}$ that has an $\mathrm{S} / \mathrm{N}$ of $3.9 \mathrm{in}$ the $V$ data, which is not high enough to formally accept it.

Oo 2752. Only one frequency just reaches the 4.0 S/N level in the $V$ data of Oo 2752, namely, $f=5.991$ day $^{-1}$. However, because of its closeness to $6 \mathrm{day}^{-1}$, the $\mathrm{S} / \mathrm{N}$ level of the peak, and the absence of proof in the other filters, we do not accept this frequency.

\section{ADDITIONAL SEISMIC RESULTS}

\subsection{Relations between Pulsation and Basic Stellar Parameters}

In this section, we do not consider star Oo 2371, since it is an ellipsoidal binary and not a pulsating star. We also exclude Oo 2086 and Oo 2633, which are, according to their frequency spectra, $\delta$ Sct stars rather than members of the cluster. This also means that their deduced position in the H-R diagram of Section 3 cannot be trusted. All other $62 \mathrm{~B}$ stars that have at least one accepted frequency in Table 4 can be considered members of NGC 884 based on the photometric diagrams presented in Appendix A of Saesen et al. (2010). Slesnick et al. (2002), Uribe et al. (2002), and Currie et al. (2010) also investigated the membership of (some) of these periodic B stars in detail, and all of them are believed to be a member star of NGC 884 by at least one of these sources.

In Figure 5 we show the frequency with the highest amplitude for every star with respect to the basic stellar parameters, 

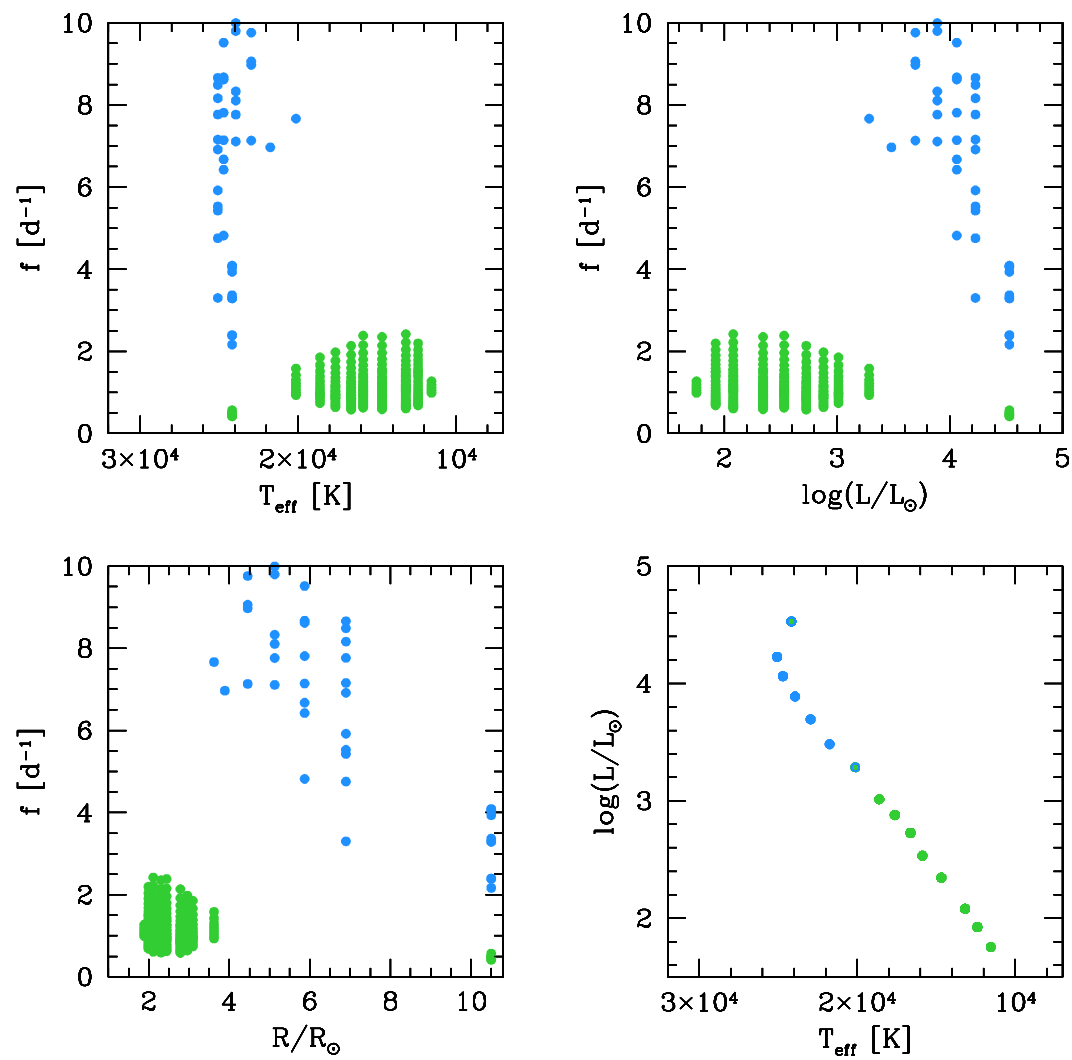

Figure 6. Same as Figure 5, but for the excited theoretical eigenfrequencies of axisymmetric modes of degree $\ell=0,1$, and 2 calculated for CLÉs models with $X=0.70, Z=0.02, \alpha_{\mathrm{ov}}=0.2$, and $\log (\mathrm{age} / \mathrm{yr})=7.1$. The green points denote the $g$-modes, the blue points the $p$-modes.

$\log \left(L / L_{\odot}\right), T_{\text {eff }}$, and the radius $R$ of the star. The latter was determined as $\left(R / R_{\odot}\right)^{2}=\left(L / L_{\odot}\right)\left(T_{\text {eff }} / T_{\text {eff, } \odot}\right)^{-4}$. We attributed different colors to different apparent groups in these diagrams, especially on the basis of the radius. We used these figures to make a selection of the most appropriate stars for asteroseismology by visual inspection. Figure 6 shows the theoretical analogue of the observational Figure 5 for axisymmetric modes and is based on CLÉs models with parameters $X=0.70, Z=0.02$, $\alpha_{\mathrm{ov}}=0.2$, and $\log (\mathrm{age} / \mathrm{yr})=7.1$ (Southworth et al. 2004b). We selected all theoretical eigenfrequencies with degrees $\ell=0,1$, and 2 for $p$ - and $g$-modes that are excited (a lower $Z$ would result in less excited frequencies). In this way, we identify the blue observational group as $p$-mode pulsators and the green one as $g$-mode pulsators. The group in between, denoted in orange, contains all Be pulsators among the studied B stars. The stars denoted in red also reside between the $p$ - and $g$-modes, but these are not known as Be stars, nor do we know their rotational velocities. On the basis of this comparison between observational and theoretical frequencies, we identify eight $\beta$ Cep pulsators in the cluster: Oo 2246, Oo 2299, Oo 2444, Oo 2488, Oo 2520, Oo 2572, Oo 2601, and Oo 2694.

In Figure 7 we show the amplitudes in the $V$ filter of all observed frequencies for all B stars with respect to the projected rotational velocities of Strom et al. (2005), Huang \& Gies (2006), and Marsh Boyer et al. (2012) for all stars for which these data are available. Stankov \& Handler (2005) found that slowly rotating Galactic $\beta$ Cep stars tend to have higher pulsation amplitudes, lending support to the hypothesis that rotation would act as an amplitude limiting mechanism. We do not find a clear connection between the observed rotation velocities and the mode amplitudes. We also note that our observed amplitudes are in general lower than the ones of the stars treated in Stankov \& Handler (2005). We do not find any dependency either between the projected rotational velocities and observed frequencies, as shown in Figure 8, but the $V$ amplitude seems to get lower for higher frequencies, as shown in Figure 9.

\subsection{Asteroseismology of the B Stars in the Cluster}

We subsequently conducted a comparison between the observed frequencies of the eight selected $\beta$ Cep stars and those that occur for our dense full grid of stellar models, to see whether we can find a consistent cluster solution, in the sense of demanding a similar chemical composition and age for the eight $\beta$ Cep stars without specifying any values for these quantities. In this way, we present a proof-of-concept for asteroseismology of the cluster without imposing information already deduced in the literature. This is useful as an independent cluster analysis because none of the previous studies allowed for a broad range in metallicity and core overshooting in their models, due to which the age uncertainties reported for the cluster in the literature are serious underestimations, as already pointed out by Southworth et al. (2004b). We have to restrict our asteroseismic analysis to the $p$-mode pulsators ( $\beta$ Cep stars) of the cluster and are not able to include the $g$-mode pulsators (SPB stars). The reason for this restriction is the dense theoretical oscillation spectrum for their $g$-modes, which we cannot exploit as long as mode identification is lacking. Indeed, given the rotation rate of the cluster stars, the predicted $g$-mode multiplets of the SPB stars are merged, and we can always assign several of them to the observed frequencies. Hence, they do not contribute to a better restriction of the cluster age, unless we can pinpoint a unique $(\ell, m)$-value to each of the detected frequencies. This is also the reason why seismic modeling of SPB stars is essentially lacking in the literature, with the notable exception of the star HD 50230 


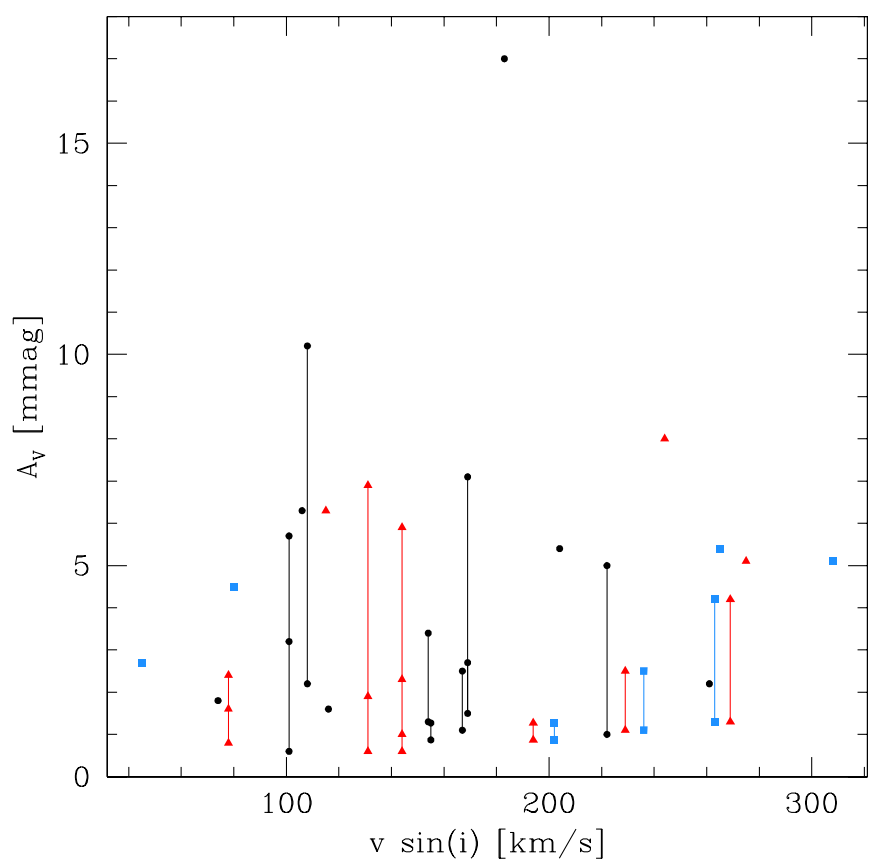

Figure 7. Amplitude in the $V$ filter of all observed frequencies with respect to the projected rotational velocities of Strom et al. (2005; circles), Huang \& Gies (2006; triangles), and Marsh Boyer et al. (2012; squares) for all stars for which these data are available. The amplitudes originating from the same star are connected.

(A color version of this figure is available in the online journal.)

in Degroote et al. (2010), for which period spacings of detected $g$-modes in this very slow rotator could be exploited in terms of input physics of the models.

In a first step, we searched for agreement between the observed and theoretical frequency spectrum on a star-to-star basis. For each of the eight $\beta$ Cep stars, we retained the grid models within the observed position in the H-R diagram within a $2 \sigma$ error box, as derived in Section 3. For these models, we made a further downselection by demanding that each observed frequency must be present in the theoretical frequency spectrum. To do this selection, we took an allowed difference between the theoretical frequency and the observed one of $\Delta f=0.15 \mathrm{day}^{-1}$ for the radial modes, because this value is half of the maximum difference in frequency for the same mode of subsequent models on the considered evolutionary tracks. For the non-radial modes, the allowed difference between the observed frequency and the theoretical one is the maximum of $\Delta f=0.15$ day $^{-1}$ or the one based on rotational splitting in the first-order approximation. Since the theoretical frequency spectra only contain axisymmetric modes, we took as the maximum allowed frequency difference for the non-radial modes of degree $\ell$ a value of $\Delta f=\ell\left(\beta_{n, l} v \sin i\right) /(2 \pi R \sin i)$, with $\beta_{n, l}$ connected with the Ledoux constant, $R$ the radius of the star (both of which are known for each model), and $v \sin i$ the projected rotational velocity. For the latter, we used the literature values given by Strom et al. (2005), Huang \& Gies (2006), or Marsh Boyer et al. (2012) if available (ranging from 45 to $144 \mathrm{~km} \mathrm{~s}^{-1}$ ), or its mean value, if more than one value is available. For two stars we did not have any estimated projected rotational velocity, and we adopted $100 \mathrm{~km} \mathrm{~s}^{-1}$ to compute $\Delta f$, but we repeated the model selection experiment taking $v \sin i=50 \mathrm{~km} \mathrm{~s}^{-1}$ and $150 \mathrm{~km} \mathrm{~s}^{-1}$ to evaluate this choice for the two stars on the outcome. We performed the downselection twice, for two values of the inclination angle, $i=30^{\circ}$ and $i=60^{\circ}$, and we restricted

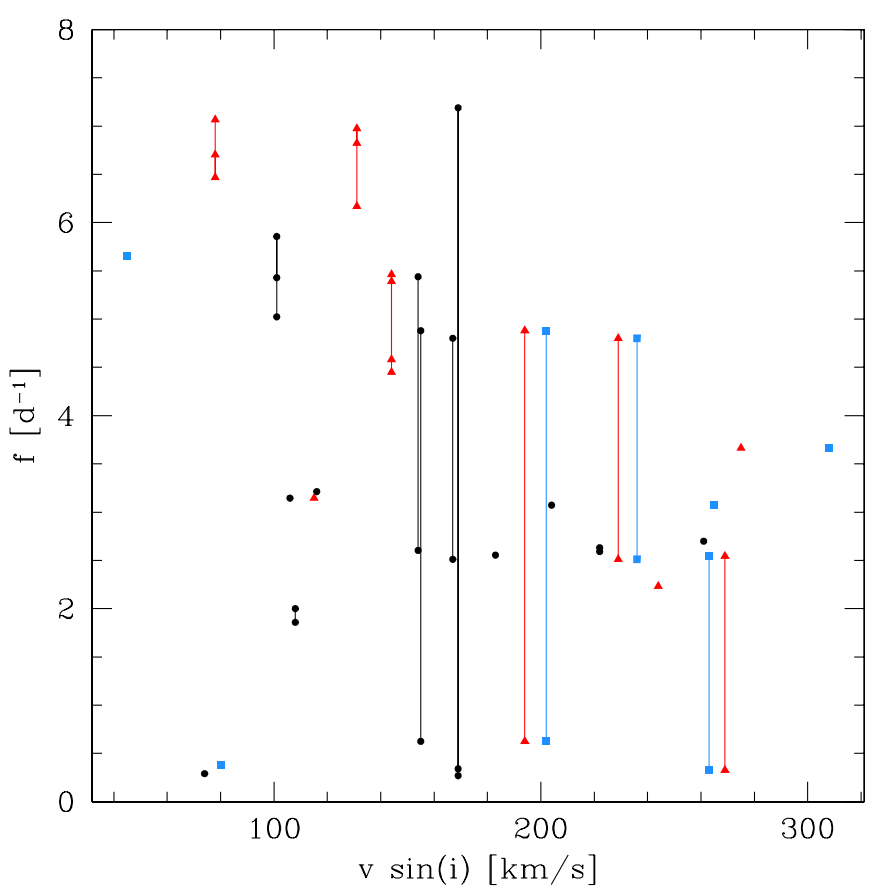

Figure 8. All observed frequency values with respect to the projected rotational velocities of Strom et al. (2005; circles), Huang \& Gies (2006; triangles), and Marsh Boyer et al. (2012; squares) for all stars for which these data are available. The frequencies observed in the same star are connected.

(A color version of this figure is available in the online journal.)

the analysis to radial modes and non-radial modes of $\ell=1$ or 2 in each case.

Since the mode identification performed in Section 5 was not conclusive for all the 19 detected frequencies in the eight selected stars, we proceeded in two different ways.

In a first scenario, the model selection done after eliminating all models outside the $2 \sigma$ error boxes in the H-R diagram only made use of the frequencies corresponding to a unique identification of $\ell$ from the amplitude ratios in Section 5, restricting to frequencies of $\ell=0,1,2$ modes. This type of model selection with one unique $\ell \leqslant 2$ per observed frequency, allowing rotational splitting, concerns seven frequencies in five $\beta$ Cep stars: Oo 2246 with $\ell=2$ for $f_{1}$ and a radial mode for $f_{3}$, Oo 2299 with $\ell=2$ for $f_{1}$, Oo 2444 with $\ell=1$ for $f_{1}$, Oo 2488 with $\ell=2$ for $f_{1}$, and Oo 2572 with $\ell=2$ for $f_{1}$ and $\ell=1$ for $f_{2}$. Since the cluster stars must have similar chemical composition and age, we only retained models with equal $(X, Z$, age) for the five stars. For each $(X, Z)$ combination, a series of models indeed survives these requirements. These selected models have an overall age range of $\log (\mathrm{age} / \mathrm{yr})=7.12-7.28$, where the lowest values occur for models with $Z=0.018$ and the highest values for those with $Z=0.010$, per fixed $X$ (see Table 5). It is noteworthy that Southworth et al. (2004b) cite an age problem, i.e., they deduced a too low range of $\log ($ age $/ y r)=7.09-7.11$ from models with fixed $Z=0.02$ and without overshoot, while they also quote the observed metallicity to be $Z \simeq 0.01$. We consider $Z<0.014$ as unlikely for the cluster, since no modes are predicted to be excited at such low metallicity for a solar mixture, but we refrain from imposing this constraint to narrow down the allowed age range at present. We looked whether the model elimination implied a restriction on the overshoot parameter for some of the five $\beta$ Cep stars, by taking their observed frequency spectrum and the obtained cluster age range into account. The only restriction on the overshoot parameters found in this way is $\alpha_{\mathrm{ov}} \geqslant 0.20$ for Oo 2299 . We currently have 
Table 5

Results of the Elimination of Models from the Grid, for Scenario 1 (Defined in the Text)

\begin{tabular}{|c|c|c|c|c|c|c|c|}
\hline \multirow[b]{2}{*}{$X$} & \multirow[b]{2}{*}{$Z$} & \multicolumn{2}{|c|}{$v \sin i=50 \mathrm{~km} \mathrm{~s}^{-1}$ for Oo 2572} & \multicolumn{2}{|c|}{$v \sin i=100 \mathrm{~km} \mathrm{~s}^{-1}$ for Oo 2572} & \multicolumn{2}{|c|}{$v \sin i=150 \mathrm{~km} \mathrm{~s}^{-1}$ for Oo 2572} \\
\hline & & $\begin{array}{c}i=30^{\circ} \\
\text { age range }\end{array}$ & $\begin{array}{c}i=60^{\circ} \\
\text { age range }\end{array}$ & $\begin{array}{c}i=30^{\circ} \\
\text { age range }\end{array}$ & $\begin{array}{c}i=60^{\circ} \\
\text { age range }\end{array}$ & $\begin{array}{c}i=30^{\circ} \\
\text { age range }\end{array}$ & $\begin{array}{c}i=60^{\circ} \\
\text { age range }\end{array}$ \\
\hline 0.68 & 0.010 & $\cdots$ & $\ldots$ & $16.32-17.84$ & $\ldots$ & $15.20-17.84$ & $16.32-16.88$ \\
\hline 0.68 & 0.012 & $17.44-17.52$ & $\cdots$ & $15.28-17.52$ & $\cdots$ & $14.72-17.52$ & $15.36-16.64$ \\
\hline 0.68 & 0.014 & $16.40-17.20$ & $\cdots$ & $14.72-17.20$ & $15.84-16.32$ & $13.84-17.20$ & $15.20-16.32$ \\
\hline 0.68 & 0.016 & $15.76-17.12$ & $\cdots$ & $13.84-17.12$ & $15.20-16.00$ & $13.36-17.12$ & $14.56-16.00$ \\
\hline 0.68 & 0.018 & $15.12-17.28$ & $\cdots$ & $13.68-17.28$ & $15.04-15.84$ & $13.20-17.28$ & $13.76-15.84$ \\
\hline 0.70 & 0.010 & $\ldots$ & $\ldots$ & $16.80-18.24$ & $\ldots$ & $15.60-18.24$ & $16.80-17.28$ \\
\hline 0.70 & 0.012 & $17.44-17.84$ & $\cdots$ & $15.84-17.84$ & $\ldots$ & $14.72-17.84$ & $15.84-16.72$ \\
\hline 0.70 & 0.014 & $16.72-17.68$ & $\cdots$ & $15.12-17.68$ & $16.32-16.64$ & $14.32-17.68$ & $15.20-16.64$ \\
\hline 0.70 & 0.016 & $15.76-17.92$ & $\ldots$ & $14.32-17.92$ & $15.68-16.32$ & $13.76-17.92$ & $15.04-16.32$ \\
\hline 0.70 & 0.018 & $15.60-17.60$ & $16.56-16.64$ & $14.08-17.60$ & $15.04-16.64$ & $13.28-17.60$ & $14.16-16.64$ \\
\hline 0.72 & 0.010 & $\ldots$ & $\cdots$ & $16.80-18.72$ & $\ldots$ & $16.08-18.72$ & $17.28-17.60$ \\
\hline 0.72 & 0.012 & 18.24 & $\ldots$ & $16.32-18.24$ & 17.28 & $15.20-18.24$ & $16.32-17.28$ \\
\hline 0.72 & 0.014 & $17.20-18.48$ & $\cdots$ & $15.60-18.48$ & $\ldots$ & $14.56-18.48$ & $15.60-16.80$ \\
\hline 0.72 & 0.016 & $16.24-18.48$ & $\cdots$ & $15.04-18.48$ & $16.16-16.64$ & $13.92-18.48$ & $15.04-16.64$ \\
\hline 0.72 & 0.018 & $16.08-18.16$ & $17.28-17.44$ & $14.24-18.16$ & $15.52-17.44$ & $13.68-18.16$ & $14.64-17.44$ \\
\hline 0.74 & 0.010 & $\cdots$ & $\cdots$ & $17.28-19.12$ & $\cdots$ & $16.56-19.12$ & $17.76-18.00$ \\
\hline 0.74 & 0.012 & 18.80 & $\cdots$ & $16.56-18.80$ & $\cdots$ & $15.68-18.80$ & $17.04-17.68$ \\
\hline 0.74 & 0.014 & $17.68-19.20$ & $\ldots$ & $15.68-19.20$ & $17.12-17.20$ & $14.80-19.20$ & $16.16-17.20$ \\
\hline 0.74 & 0.016 & $16.96-18.88$ & $\cdots$ & $15.44-18.88$ & $16.64-17.44$ & $14.24-18.88$ & $15.52-17.44$ \\
\hline 0.74 & 0.018 & $16.08-18.72$ & $17.76-18.24$ & $14.64-18.72$ & $16.00-18.24$ & $14.08-18.72$ & $15.04-18.24$ \\
\hline
\end{tabular}

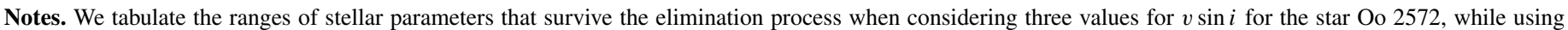

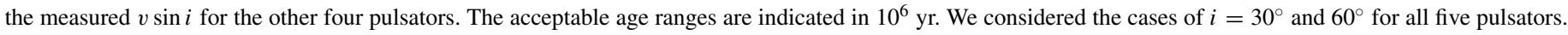
Blank entries imply that none of the models in the grid fulfill the mode identification of the detected frequencies.

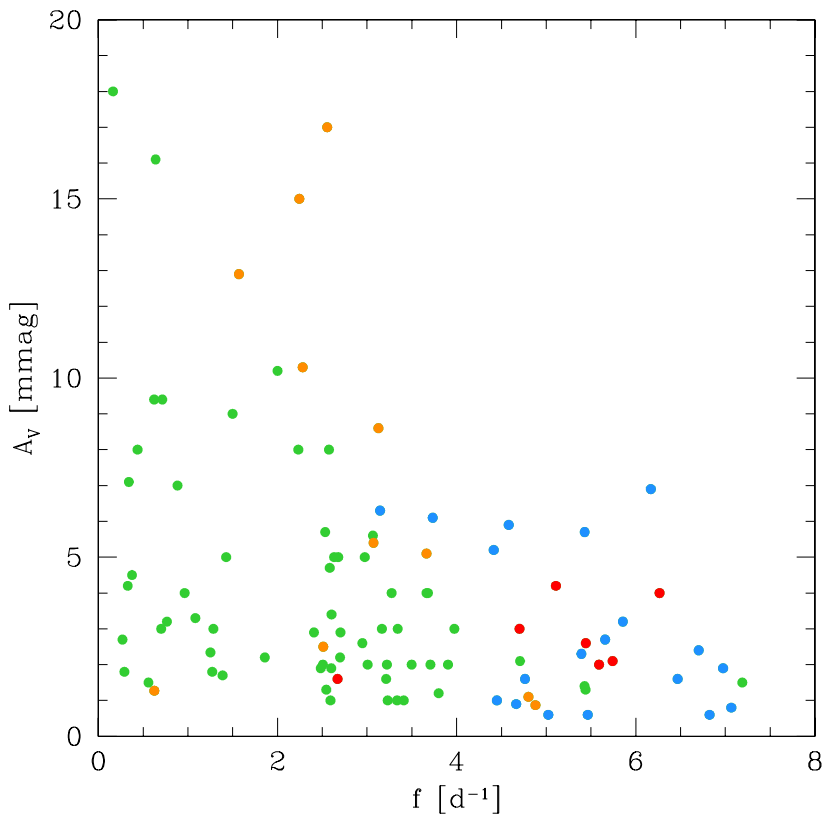

Figure 9. All observed frequency values with respect to their $V$ amplitude. For clarity we left out two points situated at $\left(f, A_{V}\right)=\left(0.20871 \mathrm{~d}^{-1}, 56 \mathrm{mmag}\right)$ and $\left(f, A_{V}\right)=\left(11.951 \mathrm{~d}^{-1}, 1 \mathrm{mmag}\right)$. The used color code is the same as for Figure 5 and is explained in Section 7.1.

insufficient observational constraints to disentangle the different physical phenomena causing near-core extra mixing, such as rotational, diffusive, or convective mixing (for a discussion of those phenomena, see Maeder 2009). Hence, despite the fact that rotational mixing leaves a different signature than plain overshoot in $\beta$ Cep stars (Montalbán et al. 2008; Miglio et al. 2008), our estimation of $\alpha_{\mathrm{ov}}$ is not sufficient to unravel the physical nature of the mixing processes at this stage.
In a second scenario, we did not make any model selection based on the identification of the degree $\ell$ of the oscillation modes as obtained from the amplitude ratios. Rather, we kept all models fulfilling the requirement of equal $(X, Z$, age) while having $\ell=0,1,2$ modes whose frequencies differ less than $\Delta f$ as determined above for the 19 detected ones in the eight stars. Again, a series of models survives these requirements, having an age range of $\log (\mathrm{age} / \mathrm{yr})=7.09-7.30$, i.e., this is less demanding on the model selection than using the seven identified frequencies as in scenario 1 . The comparison between the results of both scenarios is an illustration of the advantage of having a unique identification of the mode degree, even if only for a few modes, compared to the case where no such identification is available.

\section{SUMMARY AND DISCUSSION}

In this paper, we investigated in detail 75 periodic $B$ stars in NGC 884. The estimates for the basic stellar parameters can be found in Tables 1, 2, and 3. From a detailed frequency analysis, we deduced 65 periodic B stars, of which we classified 36 as mono-periodic, 16 as bi-periodic, 10 as tri-periodic, and 2 as quadru-periodic, while one star showed nine independent frequencies. An overview of the different frequency values, together with their amplitudes and phases from a multi-frequency fit, can be found in Table 4.

For all the detected frequencies we performed a photometric mode identification based on the comparison between the observed and the theoretical amplitude ratios, ignoring the rotational effects on the amplitude ratios. Given the input physics of the models, we could eliminate some degree possibilities. However, the large uncertainties on the observed amplitude ratios hampered a unique mode identification for the majority of detected frequencies. Table 4 contains the results for the mode 
identification in its last column. Twelve modes of nine pulsators could be securely identified.

We did not find a correlation between the projected rotational velocity and the amplitude or frequency of the modes. The amplitudes of the oscillations, however, decrease as the frequency values increase. A comparison between the observed and theoretical frequency-radius relation allowed us to select eight $\beta$ Cep stars in our sample, namely, Oo 2246, Oo 2299, Oo 2444, Oo 2488, Oo 2520, Oo 2572, Oo 2601, and Oo 2694. The seismic properties of these $\beta$ Cep stars were confronted with those predicted from an extensive grid of stellar models by requesting common cluster parameters, i.e., equal age and initial chemical composition. Elimination of models in the considered grid led to a global cluster age of $\log (\mathrm{age} / \mathrm{yr})=7.12-7.28$ by imposing the pulsation characteristics of five of the eight $\beta$ Cep stars with in total seven identified modes. This is fully compatible with the age estimate obtained from modeling of an eclipsing binary in the cluster ( $>12.6 \mathrm{Myr}$, Southworth et al. 2004b) and illustrates that the concept of cluster asteroseismology offers a valuable alternative to isochrone fitting or eclipsing binary modeling, particularly if one keeps in mind that our seismic approach considered a much broader range of $\left(X, Z, \alpha_{\mathrm{ov}}\right.$, age) than the previous methods.

If we would have had several more identified $p$-mode frequencies per star, then the elimination of models would become much more powerful. Although we have many more pulsating B stars with high-order $g$-modes at hand, they are currently not useful to restrict the seismic cluster age further, as already explained above. Ideally, one would want to reach the stage of having very detailed information on several modes, as in, e.g., Dziembowski \& Pamyatnykh (2008), Briquet et al. (2007), and Aerts et al. (2011), but then for several cluster pulsators. This requires clear detections of multiplets or high-resolution spectroscopy in addition to multi-color photometry to identify the modes. If such a stage could be reached, then the input physics of the models can be improved and a very precise cluster age could be deduced. In our present study, we only reached the stage of finding an age consistent with the one reported in the literature.

Future improvements of our present cluster modeling can be achieved by collecting high-precision spectroscopy for the cluster pulsators, in an attempt to derive usable spectroscopic limits on the metallicity. Additionally, the gathering of a time series of high-resolution high-S/N spectra of the brightest highamplitude $p$-mode pulsators in the cluster may open the door for spectroscopic mode identification of some of the oscillation modes through an interpretation of the line-profile variability (e.g., Chapter 6 in Aerts et al. 2010).

In addition to the conclusion we reached for the particular case of NGC 884, several conclusions can be drawn as best practices for further studies of this kind. A prime concern remains the overcoming of frequency aliasing and the accompanying high demands on the duty cycle of the data, particularly when the amplitudes of the cluster pulsators are of order mmag or lower. Secondly, the need occurs to have more precise amplitude determinations for secure identification of mode degrees, which was, in our study, severely hampered by the lack of a highprecision amplitude in the UV domain. This wavelength range had deserved more attention in the planning of the observing runs than we gave it.

The authors are much indebted to Prof. A. Pigulski and Dr. J. De Ridder for valuable comments on an earlier version of this study, in the framework of their PhD jury evaluation task. We thank R. Scuflaire and M.-A. Dupret for the use of their software. The research leading to these results has received funding from the European Research Council under the European Community's Seventh Framework Programme (FP7/2007-2013)/ERC grant agreement No. 227224 (PROSPERITY). This research has made use of the WEBDA database, operated at the Institute for Astronomy of the University of Vienna, as well as NASA's Astrophysics Data System, and the SIMBAD database and VizieR catalogue access tool, both operated at CDS, Strasbourg, France.

\section{REFERENCES}

Aerts, C. 2000, A\&A, 361, 245

Aerts, C., Briquet, M., Degroote, P., Thoul, A., \& van Hoolst, T. 2011, A\&A, 534, A98

Aerts, C., Christensen-Dalsgaard, J., \& Kurtz, D. W. 2010, Asteroseismology (Heidelberg: Springer)

Aerts, C., De Cat, P., Cuypers, J., et al. 1998, A\&A, 329, 137

Aerts, C., Lamers, H. J. G. L. M., \& Molenberghs, G. 2004, A\&A, 418, 639

Arenou, F., Grenon, M., \& Gomez, A. 1992, A\&A, 258, 104

Asplund, M., Grevesse, N., \& Sauval, A. J. 2005, in ASP Conf. Ser. 336, Cosmic Abundances as Records of Stellar Evolution and Nucleosynthesis, ed. T. G. Barnes III \& F. N. Bash (San Francisco, CA: ASP), 25

Ausseloos, M., Aerts, C., Lefever, K., Davis, J., \& Harmanec, P. 2006, A\&A, 455,259

Balona, L. A., Dziembowski, W. A., \& Pamyatnykh, A. 1997, MNRAS, 289, 25

Balona, L. A., Pigulski, A., Cat, P. D., et al. 2011, MNRAS, 413, 2403

Breger, M., Stich, J., Garrido, R., et al. 1993, A\&A, 271, 482

Briquet, M., Aerts, C., Baglin, A., et al. 2011, A\&A, 527, A112

Briquet, M., Morel, T., Thoul, A., et al. 2007, MNRAS, 381, 1482

Briquet, M., Uytterhoeven, K., Morel, T., et al. 2009, A\&A, 506, 269

Currie, T., Hernandez, J., Irwin, J., et al. 2010, ApJS, 186, 191

Daszyńska-Daszkiewicz, J., Dziembowski, W. A., Pamyatnykh, A. A., \& Goupil, M.-J. 2002, A\&A, 392, 151

De Cat, P., Briquet, M., Aerts, C., et al. 2007, A\&A, 463, 243

De Cat, P., Briquet, M., Daszyńska-Daszkiewicz, J., et al. 2005, A\&A, 432, 1013

De Ridder, J., Telting, J. H., Balona, L. A., et al. 2004, MNRAS, 351, 324

Degroote, P., Aerts, C., Baglin, A., et al. 2010, Natur, 464, 259

Degroote, P., Briquet, M., Catala, C., et al. 2009, A\&A, 506, 111

Desmet, M., Briquet, M., Thoul, A., et al. 2009, MNRAS, 396, 1460

Dupret, M.-A. 2002, PhD thesis, Univ. de Liège

Dupret, M.-A., De Ridder, J., De Cat, P., et al. 2003, A\&A, 398, 677

Dupret, M.-A., De Ridder, J., Neuforge, C., Aerts, C., \& Scuflaire, R. 2002, A\&A, 385, 563

Dziembowski, W. A., \& Pamyatnykh, A. A. 2008, MNRAS, 385, 2061

Flower, P. J. 1996, ApJ, 469, 355

Handler, G., Jerzykiewicz, M., Rodríguez, E., et al. 2006, MNRAS, 365, 327

Handler, G., Matthews, J. M., Eaton, J. A., et al. 2009, ApJL, 698, L56

Handler, G., \& Meingast, S. 2011, A\&A, 533, A70

Handler, G., Shobbrook, R. R., Jerzykiewicz, M., et al. 2004, MNRAS, 347,454

Handler, G., Shobbrook, R. R., \& Mokgwetsi, T. 2005, MNRAS, 362, 612

Handler, G., Shobbrook, R. R., Uytterhoeven, K., et al. 2012, MNRAS, 424, 2380

Handler, G., Shobbrook, R. R., Vuthela, F. F., et al. 2003, MNRAS, 341, 1005

Handler, G., Tuvikene, T., Lorenz, D., et al. 2008, CoAst, 157, 315

Hekker, S., Basu, S., Stello, D., et al. 2011, A\&A, 530, A100

Heynderickx, D., Waelkens, C., \& Smeyers, P. 1994, A\&AS, 105, 447

Huang, W., \& Gies, D. R. 2006, ApJ, 648, 591

Jerzykiewicz, M., Kopacki, G., Pigulski, A., Kołaczkowski, Z., \& Kim, S.-L. 2011, AcA, 61, 247

Krzesiński, J. 1998, in ASP Conf. Ser. 135, A Half Century of Stellar Pulsation Interpretation, ed. P. A. Bradley \& J. A. Guzik (San Francisco, CA: ASP), 157

Krzesiński, J., \& Pigulski, A. 1997, A\&A, 325, 987

Kunzli, M., North, P., Kurucz, R. L., \& Nicolet, B. 1997, A\&AS, 122, 51

Lenz, P., \& Breger, M. 2005, CoAst, 146, 53

Maeder, A. 2009, Physics, Formation and Evolution of Rotating Stars (Heidelberg: Springer)

Majewska, A., Pigulski, A., \& Rucinski, S. M. 2008, CoAst, 157, 338 
Malchenko, S. L. 2007, in 14th Young Scientists Conference on Astronomy and Space Physics, ed. G. Ivashchenko \& A. Golovin (Kyiv: Kyivskyi Universytet), 59

Marsh Boyer, A. N., McSwain, M. V., Aragona, C., \& Ou-Yang, B. 2012, AJ, 144,158

Meylan, G., \& Hauck, B. 1981, A\&AS, 46, 281

Michalska, G., Pigulski, A., Stęlicki, M., \& Narwid, A. 2009, AcA, 59, 349

Miglio, A., Brogaard, K., Stello, D., et al. 2012, MNRAS, 419, 2077

Miglio, A., Montalbán, J., Eggenberger, P., \& Noels, A. 2008, AN, 329, 529

Montalbán, J., Miglio, A., Eggenberger, P., \& Noels, A. 2008, AN, 329, 535

Montgomery, M. H., \& O’Donoghue, D. 1999, DSSN, 13, 28

Morel, T., Butler, K., Aerts, C., Neiner, C., \& Briquet, M. 2006, A\&A, 457, 651

Percy, J. R. 1972, PASP, 84, 420

Pigulski, A., Handler, G., Michalska, G., et al. 2007, CoAst, 150, 191

Poretti, E., \& Zerbi, F. 1993, A\&A, 268, 369

Rufener, F. 1964, PGenA, 66, 413

Rufener, F. 1985, in IAU Symp. 111, Calibration of Fundamental Stellar Quantities, ed. D. S. Hayes, L. E. Pasinetti, \& A. G. D. Philip (Cambridge: Cambridge Univ. Press), 253

Saesen, S., Carrier, F., Pigulski, A., et al. 2009, CoAst, 158, 179
Saesen, S., Carrier, F., Pigulski, A., et al. 2010, A\&A, 515, A16 Saesen, S., Pigulski, A., Carrier, F., et al. 2008, JPhCS, 118, 012071 Schwarzenberg-Czerny, A. 1991, MNRAS, 253, 198

Scuflaire, R., Montalbán, J., Théado, S., et al. 2008a, Ap\&SS, 316, 149

Scuflaire, R., Théado, S., Montalbán, J., et al. 2008b, Ap\&SS, 316, 83

Seaton, M. J. 2005, MNRAS, 362, L1

Slesnick, C. L., Hillenbrand, L. A., \& Massey, P. 2002, ApJ, 576, 880

Southworth, J., Maxted, P. F. L., \& Smalley, B. 2004a, MNRAS, 349, 547

Southworth, J., Zucker, S., Maxted, P. F. L., \& Smalley, B. 2004b, MNRAS, 355,986

Stankov, A., \& Handler, G. 2005, ApJS, 158, 193

Stello, D., Huber, D., Kallinger, T., et al. 2011, ApJL, 737, L10

Strom, S. E., Wolff, S. C., \& Dror, D. H. A. 2005, AJ, 129, 809

Telting, J. H., Abbott, J. B., \& Schrijvers, C. 2001, A\&A, 377, 104

Telting, J. H., \& Schrijvers, C. 1998, A\&A, 339, 150

Torres, G. 2010, AJ, 140, 1158

Uribe, A., García-Varela, J.-A., Sabogal-Martínez, B.-E., Higuera, G., M. A., \& Brieva, E. 2002, PASP, 114, 233

Waelkens, C., Lampens, P., Heynderickx, D., et al. 1990, A\&AS, 83, 11 